\title{
Exact Model Reduction by a Slow-Fast Decomposition of Nonlinear Mechanical Systems
}

\author{
George Haller*and Sten Ponsioen
}

November 21, 2016

Institute for Mechanical Systems, ETH Zürich

Leonhardstrasse 21, 8092 Zürich, Switzerland

\begin{abstract}
We derive conditions under which a general nonlinear mechanical system can be exactly reduced to a lower-dimensional model that involves only the most flexible degrees of freedom. This Slow-Fast Decomposition (SFD) enslaves exponentially fast the stiff degrees of freedom to the flexible ones as all oscillations converge to the reduced model defined on a slow manifold. We obtain an expression for the domain boundary beyond which the reduced model ceases to be relevant due to a generic loss of stability of the slow manifold. We also find that near equilibria, the SFD gives a mathematical justification for two modal-reduction methods used in structural dynamics: static condensation and modal derivatives. These formal reduction procedures, however, are also found to return incorrect results when the SFD conditions do not hold. We illustrate all these results on mechanical examples.
\end{abstract}

\section{Introduction}

While often hoped otherwise, a typical multi-degree-of-freedom mechanical system cannot necessarily be reduced to a lower-dimensional model. There is often a good reason why the original model involves several degrees of freedom, all of which are essential to reproduce the dynamics at the required level of accuracy.

For any multi-degree-of-freedom system, projections to various linear subspaces are nevertheless routinely employed for model reduction purposes (see Besselink et al. [4] for a review of techniques in structural vibrations, Benner et al. [3] for a more general survey). Most often, however, the accuracy or even the fundamental validity of these procedures is a priori unknown. The main reason is that distinguished subspaces identified from linearization or other considerations are generally not invariant under the nonlinear dynamics. As a consequence, trajectories of the full system do not follow those of a projection-based model, as shown in Fig. 1 .

A model reduction principle can be justified in a strict mathematical sense if the reduced model is defined on an invariant set of the full nonlinear system, and hence model trajectories are actual trajectories of the full system. In addition, the invariant set carrying the model dynamics should be robust and attracting for the reduced model to be of relevance for typical trajectories. While numerical or perturbative approximations to such a set will at best be approximately invariant, the attractivity of the actual invariant manifold is expected to keep the impact of non-invariance small, driving trajectories toward the actual invariant set.

Motivated by these considerations, we propose here two requirements for mathematically justifiable and robust model reduction in a nonlinear, non-autonomous mechanical system:

\footnotetext{
${ }^{*}$ Corresponding author. Email: georgehaller@ethz.ch
} 


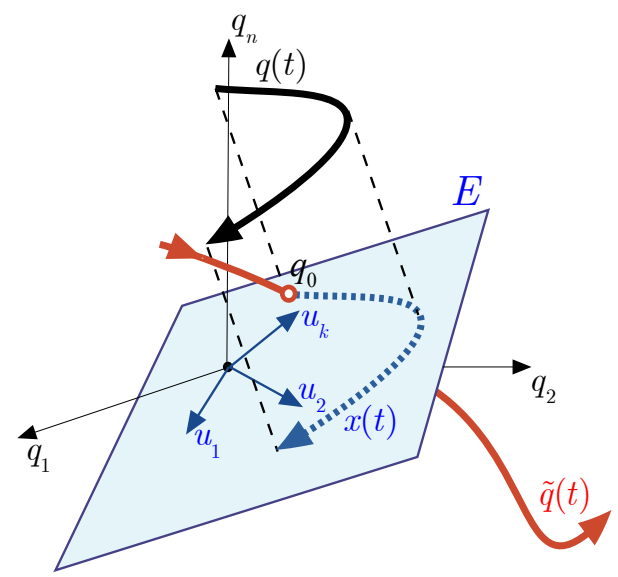

Figure 1: Model reduction by projection of a full trajectory $q(t)$ onto a $k$-dimensional subspace $E$, typically spanned by a few eigenvectors $u_{1}, \ldots, u_{k}$ of the linearized system at the origin. The model trajectory $x(t)$ starting from a point $q_{0} \in E$ is constrained to lie in $E$, but the full trajectory $\tilde{q}(t)$ starting from $q_{0}$ will generally leave the plane $E$.

(R1) There exists an attracting and persistent lower-dimensional forward-invariant manifold $\mathcal{M}(t)$. Along the manifold $\mathcal{M}(t)$, the modeled degrees of freedom (with generalized coordinates $y$ and velocities $\dot{y}$ ) are smooth functions of the modeling degrees of freedom (with generalized coordinates $x$ and velocities $\dot{x}$ ) .

(R2) General trajectories approaching $\mathcal{M}(t)$ synchronize with model trajectories at rates that are faster than typical rates within $\mathcal{M}(t)$.

By the requirement (R1), the construction of a smooth, lower-dimensional dynamical model should be equivalent to a reduction to a lower-dimensional invariant manifold, as illustrated in Fig. 22a. The dynamics on this manifold, however, is only relevant for the full system dynamics if nearby motions $q(t)$ approach model trajectories on $\mathcal{M}(t)$, i.e., the manifold has a domain of attraction foliated by stable manifolds of individual model trajectories. In addition, we require $\mathcal{M}(t)$ to be persistent (robust under small perturbations) since mechanical models have inherent parameter uncertainties and approximations, and a model reduction should be robust with respect to these.

Requirement (R2) ensures that full system trajectories not only approach the set of model trajectories in the phase space, but also synchronize with specific model trajectories. Consider, for example, a linear, two-degree of freedom mechanical system with an asymptotically stable fixed point at the origin. The fast stable manifold $\mathcal{M}$ of this fixed point (cf. Fig. 2b) is invariant, attracting and persistent, even unique (see, e.g., Cabre et al. [5]). Yet, the dynamics on $\mathcal{M}$ fails to act as a faithful reduced-order model for the typical near-equilibrium dynamics. Indeed, the flow on $\mathcal{M}$ predicts a fast decay rate that is unobservable along typical trajectories on their way to the fixed point. This is because general trajectories first approach the $(x, \dot{x})=(0,0)$ subspace, then creep towards the origin more slowly, synchronizing with motions along this subspace, rather than with those in $\mathcal{M}$.

In contrast, the invariant manifold $\overline{\mathcal{M}}$ in Fig. $2 \mathrm{p}$ satisfies both (R1) and (R2) and is indeed a good choice for model reduction. Tis is ensured by a dichotomy of time scales created by the gap in the real part of the spectrum of the eigenvalues of the fixed point. The larger this gap, the more efficient the reduced-order model in predicting typical system behavior.

In a nonlinear system, one generally loses the local slow-fast dichotomy of time scales that may arise near fixed points, such as the one in Fig. 2p. A global reduced-order model with the properties (R1)-(R2) will, therefore, not exist unless the slow-fast timescale difference created locally by the 


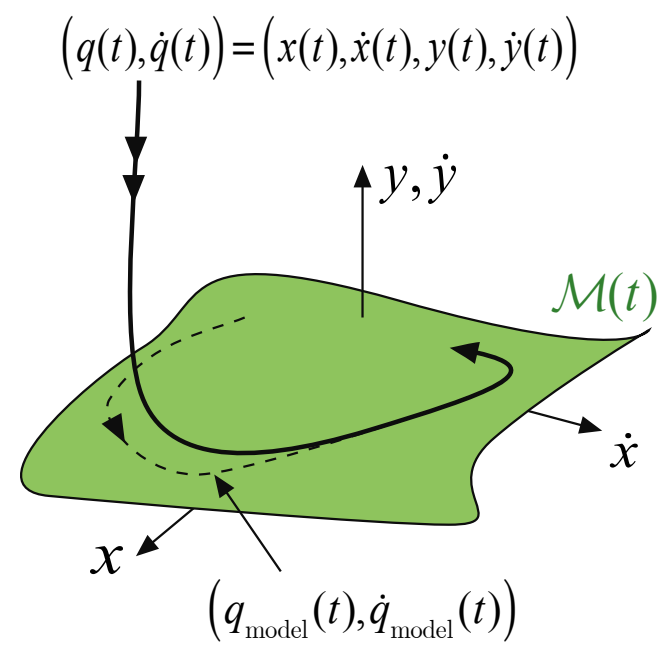

(a)

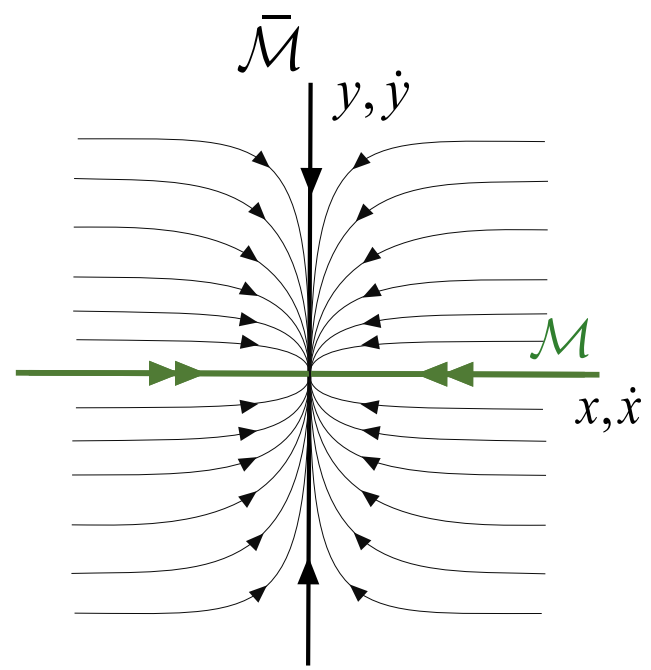

(b)

Figure 2: (a) Illustration of the geometry of requirements (R1) and (R2) for model reduction in a mechanical system with generalized coordinates $q$ and associated velocities $\dot{q}$. The reduced model depends only on a smaller group of degrees of freedom, described by the position vector $x$ and the corresponding velocity vector $\dot{x}$. The remaining degrees of freedom are characterized by the positions $y$ and velocities $\dot{y}$. (b) An attracting and persistent invariant manifold $\mathcal{M}$ that does not provide a faithful reduced-order model for the full system dynamics.

fixed point extends to a larger domain of the phase space. In more mechanical terms, a global model reduction is only feasible when the $x$ variables stay globally stiffer (i.e., faster) than the $y$ variables.

Such a global slow-fast partition of coordinates has been assumed in several case studies of mechanical systems, such as an undamped spring coupled to a pendulum (Georgiou and Schwartz [10]) and its extensions to higher or even infinitely many dimensions (Georgiou and Schwartz [13] and Georgiou and Vakakis [11). These studies tacitly assume the existence of a slow manifold without specific consideration to its stability and robustness. Due to a lack of normal hyperbolicity for the limiting slow manifold (critical manifold), well-defined invariant slow manifolds do not actually exist in these mechanical models. Recent results guarantee only near-invariant surfaces under certain conditions (MacKay [25], Kristianssen and Wullf [22]). These surfaces, however, do not attract trajectories from an open neighborhood of the phase space. As a result, their relevance for model reduction is a priori unclear, as they violate the requirement (R1).

As a further case study, a forced and stiff linear oscillator coupled to a soft nonlinear oscillator was considered by Georgiou et al. [9, 12. As the authors observe, the existence of an attracting, two-dimensional slow manifold in these two studies follows from a globalized version of the center manifold theorem (Carr [6]) and from the geometric singular perturbation formulation of Fenichel 
[8], respectively. These approaches are similar in spirit to the work we describe here, but pertain to specific, low-dimensional, soft-stiff mechanical models without targeting model reduction issues per se.

Related work also includes that of Lubich [24], who developed a numerical scheme for mechanical systems with very stiff potential forces. In this context, all degrees of freedom are equally fast and hence no oscillatory mode can be enslaved to the rest via model reduction. An exceptional slow manifold (which involves coordinates from all degrees of freedom) becomes attracting only under the numerical scheme. A numerical procedure is introduced for approximating such slow manifolds in more general but still uniformly stiff mechanical systems by Ariel et al. 1. In a more mathematical treatment, Stumpp [31] considered general mechanical systems with stiff damping forces and showed the existence of an attracting slow manifold governing the asymptotic behavior of the system. Again, all degrees of freedom are assumed equally stiff and hence no modes can be eliminated via model reduction.

In contrast to these specific case studies and purely stiff reduction procedures, we consider here general mechanical systems and establish conditions under which stiffer degrees of freedom can be identified and eliminated by reduction to an attracting slow manifold defined over the remaining softer degrees of freedom. We do not assume any specific force or inertia term to be large or small. Rather, we seek the broadest set of conditions under which an exact slow-fast decomposition emerges and yields a reduced-order mechanical system. The slow-fast decomposition (SDF) procedure arising form our analysis satisfies the key requirements (R1)-(R2) discussed above.

We also establish the maximal domain of SFD, and give a specific upper bound on the rate at which general solutions synchronize with those of the reduced-order model. Our includes several classes of mechanical systems and justifies earlier heuristic reduction schemes under certain conditions. In particular, under the SFD conditions, the techniques of static condensation and modal derivatives, respectively, can rigorously be justified as first- and second-order local approximations to a slow manifold near an equilibrium. At the same time, we give examples of these reduction procedures fail when the SFD conditions are not met.

We illustrate these results on simple mechanical systems, but our formulas are explicit enough to be applied to higher-degree-of-freedom problems. Importantly, determining the eigenvalues and modes shapes is not a prerequisite for the application of SFD. Indeed, the stiffer modes may be fully (both linearly and nonlinearly) coupled to the rest of the modes

\section{Set-up}

\subsection{General form of the mechanical system}

Consider a $n$-degree of freedom, non-dimensionalized mechanical system of the form

$$
M(q, t) \ddot{q}-F(q, \dot{q}, t)=0,
$$

where $M \in \mathbb{R}^{n \times n}$ is a nonsingular mass matrix that may depend on the generalized coordinates $q$ and the time $t$ in a smooth fashion (class $C^{r}$ for some $r \geq 2$ ). The internal and external forces acting on the system are contained in the term $F \in \mathbb{R}^{n}$, which generally depends on $q, t$ and the generalized velocities $\dot{q} \in \mathbb{R}^{n}$.

\subsection{Classic model reduction by projection to a subspace}

As noted in the Introduction, model reduction for system (1) is generally motivated by an assumed coordinate change

$$
q=U x,
$$

with a matrix $U \in \mathbb{R}^{n \times k}$ and a reduced coordinate vector $x \in \mathbb{R}^{k}$ with $k<n$ (see, e.g., Geradin and Rixen [14]). Substitution into (1), followed by a multiplication by $U^{T}$, then suggests the reduced 
equations of motion

$$
U^{T} M(U x, t) U \ddot{x}-U^{T} F(U x, U \dot{x}, t)=0,
$$

the projection of 11 from the full state space $\mathbb{R}^{n}$ onto a $k$-dimensional subspace $E$, parametrized by the variable $x$ (cf. Fig. 1). The main focus of model reduction studies is then the most expedient choice of the matrix $U$.

It is often forgotten, however, that for eq. (3) to hold, one must have $q(t)=U x(t)$ for all times, i.e., $E$ must be an invariant plane for (1). This assumption is practically certain to be violated unless special symmetries are present. Even for unforced and stable structural system (i.e., when (1) is autonomous and $q=0$ is asymptotically stable), the invariance of modal subspaces is violated when nonlinear terms are present. The mismatch between modal subspaces and (nonlinear) invariant manifolds emanating from the origin will only be small very close to the origin. In addition, various choices of $U$ may render projected equations that do not capture typical dynamics even close to $q=0$ (cf. Haller and Ponsioen [17] and Section 5] below for examples).

\subsection{Slow (flexible) and fast (stiff) variables}

If the system (1) is non-autonomous, we assume that its explicit time-dependence in $M$ and $F$ is precisely one of the following three types: (1) periodic (2) quasiperiodic with finitely many rationally independent frequencies (3) aperiodic over a finite time interval $[a, b]$. In the periodic and quasiperiodic cases, we let $t \in \mathcal{T}=\mathbb{R}$, whereas in the aperiodic case, we let $t \in \mathcal{T}=[a, b]$.

Next, we split the generalized coordinate vector $q$ as

$$
q=\left(\begin{array}{l}
x \\
y
\end{array}\right), \quad x \in \mathbb{R}^{s}, \quad y \in \mathbb{R}^{f}, \quad s+f=n,
$$

into yet unspecified slow coordinates $x$ and fast coordinates $y$. This slow-fast partition refers to the expected relative speed of variation of the $x$ and $y$ variables. In mechanical terms, we expect $x$ to label relatively flexible degrees of freedom as opposed to the relatively stiff degrees of freedoms labeled by the $y$ coordinates.

We seek conditions under which a mathematically rigorous model reduction process exists to express $y(t)$ uniquely as function of $x(t)$, at least asymptotically in time, along general trajectories $q(t)$ of (1). The $(x, y)$ partition of $q$ may be suggested by a modal analysis of the linear system or simply by the physics of a mechanical problem. Importantly, $q$ is not assumed to be a set of linear modal coordinates, and hence our procedure does not rely on an a priori identification of a linearized spectrum near an equilibrium point.

To allow for a potentially stiff dependence of the system on $y$, we introduce a small, nondimensional parameter $\epsilon>0$ and consider $M$ and $F$ as smooth functions of $y / \epsilon$ and $y$ for $\epsilon>0$. At this point, this represents no loss of generality, given that any smooth function of $y$ and $\epsilon$ can also be viewed as a smooth function of $y / \epsilon$ and $\epsilon$ for $\epsilon>0$ because $y=\epsilon \cdot(y / \epsilon)$.

Using this notation, we split the mass matrices and forcing terms in (1) by letting

$$
M(q, t)=\left(\begin{array}{cc}
M_{11}\left(x, \frac{y}{\epsilon}, t ; \epsilon\right) & M_{12}\left(x, \frac{y}{\epsilon}, t ; \epsilon\right) \\
M_{21}\left(x, \frac{y}{\epsilon}, t ; \epsilon\right) & M_{22}\left(x, \frac{y}{\epsilon}, t ; \epsilon\right)
\end{array}\right), \quad F(q, \dot{q}, t)=\left(\begin{array}{c}
F_{1}\left(x, \dot{x}, \frac{y}{\epsilon}, \dot{y}, t ; \epsilon\right) \\
F_{2}\left(x, \dot{x}, \frac{y}{\epsilon}, \dot{y}, t ; \epsilon\right)
\end{array}\right), \quad \epsilon>0,
$$

where $M_{11} \in \mathbb{R}^{s \times s}, M_{12}, M_{21}^{T} \in \mathbb{R}^{s \times f}, M_{22} \in \mathbb{R}^{f \times f}, F_{1} \in \mathbb{R}^{s}$ and $F_{2} \in \mathbb{R}^{f}$. Again, as notated above, this notation is general enough to allow for cases in which $M$ or $F$ depends purely on $y$, or depends both on $y$ and $y / \epsilon$. The corresponding equations of motion are

$$
\begin{aligned}
& M_{11} \ddot{x}+M_{12} \ddot{y}-F_{1}=0, \\
& M_{22} \ddot{y}+M_{21} \ddot{x}-F_{2}=0 .
\end{aligned}
$$


Taking appropriate linear combination of these equations, and introducing the matrix $M_{i}$ and forces $Q_{i}$ via

$$
\begin{aligned}
M_{i}\left(x, \frac{y}{\epsilon}, t ; \epsilon\right) & =M_{i i}-M_{i j} M_{j j}^{-1} M_{j i}, \quad i, j=1,2, \quad i \neq j, \\
Q_{i}\left(x, \dot{x}, \frac{y}{\epsilon}, \dot{y}, t ; \epsilon\right) & =F_{i}-M_{i j} M_{j j}^{-1} F_{j}, \quad i, j=1,2, \quad i \neq j,
\end{aligned}
$$

we deduce from (4) the inertially decoupled equations of motion:

$$
\begin{aligned}
& M_{1}\left(x, \frac{y}{\epsilon}, t ; \epsilon\right) \ddot{x}-Q_{1}\left(x, \dot{x}, \frac{y}{\epsilon}, \dot{y}, t ; \epsilon\right)=0, \\
& M_{2}\left(x, \frac{y}{\epsilon}, t ; \epsilon\right) \ddot{y}-Q_{2}\left(x, \dot{x}, \frac{y}{\epsilon}, \dot{y}, t ; \epsilon\right)=0 .
\end{aligned}
$$

Importantly, the equations (6) are fully equivalent to (1) for any choice of the partition $q=$ $(x, y)$ and for any choice of a scalar parameter $\epsilon>0$. In particular, $M_{1}\left(x, \frac{y}{\epsilon}, t ; \epsilon\right) \in \mathbb{R}^{s \times s}$ and $M_{2}\left(x, \frac{y}{\epsilon}, t ; \epsilon\right) \in \mathbb{R}^{f \times f}$ are nonsingular matrices for all $(x, y, t)$ and for all $\epsilon>0$. As we shall see in later examples, the partition $q=(x, y)$ will need to be selected in given problems in a way that further assumptions detailed below are satisfied.

\subsection{Assumptions of the SFD and illustrating examples}

We now list assumptions that will be sufficient to guarantee the existence of an exact reduced-order model satisfying the requirements (R1)-(R2). First, using the new variable $\eta=y / \epsilon$, we define the mass-normalized forcing terms

$$
\begin{aligned}
& P_{1}(x, \dot{x}, \eta, \dot{y}, t ; \epsilon)=M_{1}^{-1}(x, \eta, t ; \epsilon) Q_{1}(x, \dot{x}, \eta, \dot{y}, t ; \epsilon), \\
& P_{2}(x, \dot{x}, \eta, \dot{y}, t ; \epsilon)=\epsilon M_{2}^{-1}(x, \eta, t ; \epsilon) Q_{2}(x, \dot{x}, \eta, \dot{y}, t ; \epsilon),
\end{aligned}
$$

which are, by our assumptions, class $C^{r}$ in their arguments for $\epsilon>0$. The following assumptions concern properties of $P_{i}$ in their $\epsilon=0$ limit.

(A1) Nonsingular extension to $\epsilon=0$ : The functions $P_{1}$ and $P_{2}$ are at least of class $C^{2}$ in their arguments at $\epsilon=0$.

In other words, assumption (A1) requires continuous differentiability of the transformed forcing terms $P_{i}$ also in the limit of $\epsilon=0$, when the dummy variable $\eta=y / \epsilon$ is held fixed, independent of $\epsilon$.

(A2) Existence of a fast zero-acceleration set (critical manifold): The algebraic equation $Q_{2}(x, \dot{x}, \eta, 0, t ; 0) \equiv 0$ can be solved for $\eta$ on an open, bounded domain $\mathcal{D}_{0} \subset \mathbb{R}^{s} \times \mathbb{R}^{s} \times \mathcal{T}$. Specifically, there exists a $C^{1}$ function $G_{0}: \mathcal{D}_{0} \rightarrow \mathbb{R}^{s}$ such that

$$
Q_{2}\left(x, \dot{x}, G_{0}(x, \dot{x}, t), 0, t ; 0\right) \equiv 0
$$

holds for all $(x, \dot{x}, t) \in \mathcal{D}_{0}$. We refer to the set $\mathcal{M}_{0}(t)$ defined by $\eta=G_{0}(x, \dot{x}, t)$ as a critical manifold.

Assumption (A2) ensures the existence of a smooth set $\mathcal{M}_{0}(t)$ of instantaneous zero-acceleration states (critical manifold) for the fast coordinates. Here the velocity variable $\dot{x} \in \mathbb{R}^{s}$ is viewed as arbitrary, and hence unrelated to the actual time derivative of $x(t)$ along a trajectory $q(t)$. As a consequence, these instantaneous zero-acceleration states are not equilibria and do not form an invariant set for system (6). Under assumption (A3) below, however, $\mathcal{M}_{0}(t)$ will turn out to approximate a slow invariant manifold that carries a reduced-order model satisfying the requirements (R1)-(R2): 
(A3) Formal asymptotic stability of the critical manifold: With the matrices

$$
A(x, \dot{x}, t)=-\partial_{\dot{y}} P_{2}\left(x, \dot{x}, G_{0}(x, \dot{x}, t), 0, t ; 0\right), \quad B(x, \dot{x}, t)=-\partial_{\eta} P_{2}\left(x, \dot{x}, G_{0}(x, \dot{x}, t), 0, t ; 0\right),
$$

the equilibrium solution $\eta \equiv 0 \in \mathbb{R}^{f}$ of the unforced, constant-coefficient linear system

$$
\eta^{\prime \prime}+A(x, \dot{x}, t) \eta^{\prime}+B(x, \dot{x}, t) \eta=0
$$

is asymptotically stable for all fixed parameter values $(x, \dot{x}, t) \in \mathcal{D}_{0}$. Here prime denotes differentiation with respect to an auxiliary time $\tau$ that is independent of $t$.

Note that assumption (A3) requires the linear unforced oscillatory system (9), posed formally for the dummy fast variable $\eta$, to be asymptotically stable. In this context, $(x, \dot{x}, t)$ play the role of constant parameters ranging over $\mathcal{D}_{0}$. Assumption (A3) is satisfied, for instance, when $A$ is symmetric, positive semi-definite and $B$ is symmetric, positive definite over $\mathcal{D}_{0}$. In that case, $A$ represents a damping matrix and $B$ represents a stiffness matrix for all parameter values $(x, \dot{x}, t) \in \mathcal{D}_{0}$. At this point, (A3) is only a formal requirement with no immediately clear mathematical meaning. This is because the critical manifold $\mathcal{M}_{0}(t)$ is not invariant under equation (6) and hence the arguments of $A$ and $B$ are, in fact, time-varying, and hence do not determine the stability of (9).

Example 1. [Weakly nonlinear system with parametric forcing] Consider a typical multi-degree-offreedom mechanical system of the form

$$
\begin{aligned}
M_{1} \ddot{x}+C_{1} \dot{x}+K_{1} x+S_{1}(x, y) & =f_{1}(t), \\
M_{2} \ddot{y}+C_{2} \dot{y}+K_{2} y+S_{2}(x, y) & =f_{2}(t),
\end{aligned}
$$

with $x \in \mathbb{R}^{s}$ and $y \in \mathbb{R}^{f}$. Here the $M_{i}$ are symmetric and positive definite constant mass matrices; $C_{i}$ are constant symmetric damping matrices; $K_{i}$ are constant symmetric stiffness matrices; and the functions

$$
S_{i}(x, y)=\mathcal{O}\left(|x|^{2},|x||y|,|y|^{2}\right)
$$

model nonlinear coupling terms. By definition (5), for an arbitrary scalar parameter $\epsilon>0$ independent of $M_{i}, C_{i}, D_{i}$ and $S_{i}$, we specifically have

$$
\begin{aligned}
& Q_{1}\left(x, \dot{x}, \frac{y}{\epsilon}, \dot{y}, t ; \epsilon\right)=-\left[C_{1} \dot{x}+K_{1} x+S_{1}\left(x, \epsilon \frac{y}{\epsilon}\right)-f_{1}(t)\right] \\
& Q_{2}\left(x, \dot{x}, \frac{y}{\epsilon}, \dot{y}, t ; \epsilon\right)=-\left[C_{2} \dot{y}+\epsilon K_{2}\left(\frac{y}{\epsilon}\right)+S_{2}\left(x, \epsilon \frac{y}{\epsilon}\right)-f_{2}(t)\right] .
\end{aligned}
$$

Therefore, the functions

$$
\begin{aligned}
& P_{1}(x, \dot{x}, \eta, \dot{y}, t ; \epsilon)=-M_{1}^{-1}\left[C_{1} \dot{x}+K_{1} x+S_{1}(x, \epsilon \eta)-f_{1}(t)\right], \\
& P_{2}(x, \dot{x}, \eta, \dot{y}, t ; \epsilon)=-\epsilon M_{2}^{-1}\left[C_{2} \dot{y}+\epsilon K_{2} \eta+S_{2}(x, \epsilon \eta)-f_{2}(t)\right],
\end{aligned}
$$

are differentiable in $\epsilon$ at the the $\epsilon=0$ limit, satisfying assumption (A1). However, we have

$$
P_{2}(x, \dot{x}, \eta, \dot{y}, t ; 0) \equiv 0 .
$$

Therefore, while any function $G_{0}(x, \dot{x}, t)$ satisfies (A2), both matrices $A$ and $B$ defined in (8) vanish, and hence assumption (A3) never holds for system (10). For this assumption to hold, some of the system parameters must be related to the small parameter $\epsilon$, as we shall see in the next two examples.

Example 2. [Partially stiff weakly nonlinear system with very small stiff-inertia and parametric forcing] Consider now the slightly modified multi-degree-of-freedom mechanical system

$$
\begin{aligned}
M_{1} \ddot{x}+C_{1} \dot{x}+K_{1} x+S_{1}(x, y) & =f_{1}(t), \\
\epsilon^{2} M_{2} \ddot{y}+C_{2} \dot{y}+\frac{1}{\epsilon} K_{2} y+S_{2}(x, y) & =f_{2}(t),
\end{aligned}
$$


with a non-dimensional small parameter $\epsilon \ll 1$. All variables, matrices and functions are the same as in Example 1, but the $y$-component of this system generates very small inertial forces and also has large linear stiffness. This time, we have

$$
\begin{aligned}
& P_{1}(x, \dot{x}, \eta, \dot{y}, t ; \epsilon)=-M_{1}^{-1}\left[C_{1} \dot{x}+K_{1} x+S_{1}(x, \epsilon \eta)-f_{1}(t)\right], \\
& P_{2}(x, \dot{x}, \eta, \dot{y}, t ; \epsilon)=-\frac{1}{\epsilon} M_{2}^{-1}\left[C_{2} \dot{y}+K_{2} \eta+S_{2}(x, \epsilon \eta)-f_{2}(t)\right],
\end{aligned}
$$

therefore assumption (A1) is not satisfied, given that $P_{2}$ is not differentiable at $\epsilon=0$.

Example 3. [Paradigm for targeted energy transfer: Weakly nonlinear system with small inertia in its essentially nonlinear component] Consider the multi-degree-of-freedom mechanical system

$$
\begin{aligned}
M_{1} \ddot{x}+C_{1} \dot{x}+K_{1} x+S_{1}(x, y) & =0, \\
\epsilon M_{2} \ddot{y}+C_{2} \dot{y}+S_{2}(x, y) & =0,
\end{aligned}
$$

with a non-dimensional small parameter $\epsilon \ll 1$. Again, all variables and matrices are the same as in Example (1), but the $y$-component of (13) generates small inertial forces and no linear stiffness forces. This system is noted as a prototype example of targeted energy transfer (cf. Vakakis et al. [32]) from the $x$ degrees of freedom to the $y$ degrees of freedom. This energy transfer mechanism suggests the lack of a reduced-order model over the $x$-degrees of freedom, given that the $y$-variables display no long-term enslavement to the $x$-variables. Calculating the quantities in our assumption (A1), we find

$$
\begin{aligned}
& P_{1}(x, \dot{x}, \eta, \dot{y}, t ; \epsilon)=-M_{1}^{-1}\left[C_{1} \dot{x}+K_{1} x+S_{1}(x, \epsilon \eta)\right] \\
& P_{2}(x, \dot{x}, \eta, \dot{y}, t ; \epsilon)=-M_{2}^{-1}\left[C_{2} \dot{y}+S_{2}(x, \epsilon \eta)\right]
\end{aligned}
$$

are differentiable at $\epsilon=0$, and hence assumption (A1) holds. However, the equation

$$
Q_{2}(x, \dot{x}, \eta, 0, t ; 0)=-S_{2}(x, 0)=0
$$

cannot be solved for the variable $\eta$ at any point. As a consequence, even though a set of zero acceleration states is defined by the equation $S_{2}(x, 0)=0$, this set is not attracting. Indeed, the matrix $B(x, v, t)$ defined in assumption (A3) vanishes identically and hence the linear system (9) is not asymptotically stable.

Example 4. [Partially stiff weakly nonlinear system with parametric forcing] Consider now the multi-degree-of-freedom mechanical system

$$
\begin{aligned}
M_{1} \ddot{x}+C_{1} \dot{x}+K_{1} x+S_{1}\left(x, \frac{y}{\epsilon}\right) & =f_{1}(t), \\
\epsilon M_{2} \ddot{y}+C_{2} \dot{y}+\frac{1}{\epsilon} K_{2} y+S_{2}(x, y) & =f_{2}(t),
\end{aligned}
$$

with the same quantities as in Example (1). The difference here is that the mass matrix of the $y$ degrees of freedom has small norm for $\epsilon \ll 1$ and the stiffness matrix is large in norm in the same equation. In addition, the nonlinear coupling term in the $x$-equation is assumed to have a stiff dependence on $y$. In this case, we have

$$
\begin{aligned}
& P_{1}(x, \dot{x}, \eta, \dot{y}, t ; \epsilon)=-M_{1}^{-1}\left[C_{1} \dot{x}+K_{1} x+S_{1}(x, \eta)-f_{1}(t)\right], \\
& P_{2}(x, \dot{x}, \eta, \dot{y}, t ; \epsilon)=-M_{2}^{-1}\left[C_{2} \dot{y}+K_{2} \eta+S_{2}(x, \epsilon \eta)-f_{2}(t)\right],
\end{aligned}
$$

which satisfy assumption (A1). Solving the equation $Q_{2}(x, \dot{x}, \eta, 0, t ; 0)=0$ for $\eta$, we find that assumption (A2) is satisfied by the function

$$
G_{0}(x, \dot{x}, t)=K_{2}^{-1}\left[f_{2}(t)-S_{2}(x, 0)\right], \quad(x, \dot{x}, t) \in \mathcal{D}_{0}=\mathbb{R}^{s} \times \mathbb{R}^{s} \times \mathbb{R},
$$


provided that the stiffness matrix $K_{2}$ is invertible. In that case, we obtain

$$
A(x, \dot{x}, t)=M_{2}^{-1} C_{2}, \quad B(x, \dot{x}, t)=\left.M_{2}^{-1}\left[K_{2}+\epsilon \partial_{y} S_{2}\left(x, \epsilon G_{0}(x, \dot{x}, t)\right)\right]\right|_{\epsilon=0}=M_{2}^{-1} K_{2},
$$

and hence the homogeneous linear oscillatory system in assumption (A3) becomes

$$
\eta^{\prime \prime}+M_{2}^{-1} C_{2} \eta^{\prime}+M_{2}^{-1} K_{2} \eta=0
$$

or, equivalently,

$$
M_{2} \eta^{\prime \prime}+C_{2} \eta^{\prime}+K_{2} \eta=0 .
$$

The zero equilibrium of this system is asymptotically stable by our assumptions on $M_{2}, C_{2}$ and $K_{2}$. Therefore, assumption (A3) is also satisfied for system (14).

\section{Main result: Global existence of an exact reduced-order model}

To state our main result formally, we first define the following functions for all $(x, \dot{x}, t) \in \mathcal{D}_{0}$ :

$$
\begin{aligned}
H_{0}(x, \dot{x}, t)= & \partial_{x} G_{0}(x, \dot{x}, t) \dot{x}+\partial_{\dot{x}} G_{0}(x, \dot{x}, t) P_{1}\left(x, \dot{x}, G_{0}(x, \dot{x}, t), 0, t ; 0\right)+\partial_{t} G_{0}(x, \dot{x}, t), \\
G_{1}(x, \dot{x}, t)= & -\left[D_{\eta} P_{2}\left(x, \dot{x}, G_{0}(x, \dot{x}, t), 0, t ; 0\right)\right]^{-1} D_{\dot{y}} P_{2}\left(x, \dot{x}, G_{0}(x, \dot{x}, t), 0, t ; 0\right) H_{0}(x, \dot{x}, t) \\
& -\left[D_{\eta} P_{2}\left(x, \dot{x}, G_{0}(x, \dot{x}, t), 0, t ; 0\right)\right]^{-1} D_{\epsilon} P_{2}\left(x, \dot{x}, G_{0}(x, \dot{x}, t), 0, t ; 0\right), \\
H_{1}(x, \dot{x}, t)= & \partial_{x} G_{1}(x, \dot{x}, t) v+\partial_{\dot{x}} G_{1}(x, \dot{x}, t) P_{1}\left(x, \dot{x}, G_{0}(x, \dot{x}, t), 0, t ; 0\right)+\partial_{t} G_{1}(x, \dot{x}, t) .
\end{aligned}
$$

With these quantitates, we have the following result:

Theorem 1. Under assumptions (A1)-(A3) and for $\epsilon>0$ small enough:

(i) The mechanical system (1) admits an exact reduced-order model satisfying the requirements (R1)-(R2).

(ii) The reduced-order model is given by

$$
\begin{aligned}
\ddot{x}-P_{1}\left(x, \dot{x}, G_{0}(x, \dot{x}, t), 0, t ; 0\right)= & \epsilon\left[D_{\eta} P_{1}\left(x, \dot{x}, G_{0}(x, \dot{x}, t), 0, t ; 0\right) G_{1}(x, \dot{x}, t)\right. \\
& +D_{\dot{y}} P_{1}\left(x, \dot{x}, G_{0}(x, \dot{x}, t), 0, t ; 0\right) H_{0}(x, \dot{x}, t) \\
& \left.+D_{\epsilon} P_{1}\left(x, \dot{x}, G_{0}(x, \dot{x}, t), 0, t ; 0\right)\right] \\
+ & \mathcal{O}\left(\epsilon^{2}\right)
\end{aligned}
$$

for all $(x, \dot{x}, t) \in \mathcal{D}_{0}$.

(iii) If $M_{1}(x, \eta, \epsilon)$ is smooth in $\epsilon$ at $\epsilon=0$, then the multiplication of 18 by $M_{1}$ gives a form of the reduced-order model that does not require the inversion of $M_{1}$ :

$$
M_{1}\left(x, G_{0}(x, \dot{x}, t), t ; 0\right) \ddot{x}-Q_{1}\left(x, v, G_{0}(x, \dot{x}, t), 0, t ; 0\right)=\mathcal{O}(\epsilon) .
$$

(iv) The reduced-order models (18)-19) describe the reduced flow on a $2 s$-dimensional invariant manifold $\mathcal{M}_{\epsilon}(t)$ along which positions and velocities in the stiff degrees of freedom are enslaved to those in the slow degrees of freedom via

$$
\begin{aligned}
& y=\epsilon G_{0}(x, \dot{x}, t)+\epsilon^{2} G_{1}(x, \dot{x}, t)+\mathcal{O}\left(\epsilon^{3}\right), \\
& \dot{y}=\epsilon H_{0}(x, \dot{x}, t)+\epsilon^{2} H_{1}(x, \dot{x}, t)+\mathcal{O}\left(\epsilon^{3}\right) .
\end{aligned}
$$


(v) The $x(t)$ components of the trajectories of system (1) synchronize with appropriate model trajectories $x_{R}(t)$ of $(18)$ or $(19)$ at an exponential rate. Specifically, let $q(t)=(x(t), y(t))$ be a full trajectory of system (1) such that at a time $t_{0}$, the initial position $q\left(t_{0}\right)$ is close enough to the slow manifold carrying the reduced order model. Then there exists a trajectory $x_{R}(t)$ of the reduced-order model (18) or 190 such that

$$
\left|\left(\begin{array}{c}
x(t)-x_{R}(t) \\
\dot{x}(t)-\dot{x}_{R}(t)
\end{array}\right)\right| \leq C\left|\left(\begin{array}{c}
x\left(t_{0}\right)-x_{R}\left(t_{0}\right) \\
\dot{x}\left(t_{0}\right)-\dot{x}_{R}\left(t_{0}\right) \\
\frac{1}{\epsilon} y\left(t_{0}\right)-G_{0}\left(x_{R}\left(t_{0}\right), \dot{x}_{R}\left(t_{0}\right), t\right)+\mathcal{O}(\epsilon) \\
\dot{y}\left(t_{0}\right)-\epsilon H_{0}\left(x_{R}\left(t_{0}\right), \dot{x}_{R}\left(t_{0}\right), t\right)+\mathcal{O}\left(\epsilon^{2}\right)
\end{array}\right)\right| e^{-\frac{\Lambda}{\epsilon}\left(t-t_{0}\right),} \quad t>t_{0},
$$

where $\Lambda>0$ can be selected as any constant satisfying

$$
\max _{j \in[1, f],(x, \dot{x}, t) \in \mathcal{D}_{0}} \operatorname{Re} \lambda_{j}(x, \dot{x}, t)<-\Lambda<0,
$$

with $\lambda_{j}(x, \dot{x}, t), j=1, \ldots, f$, denoting the eigenvalues of the associated linear system 75 . The constant $C>0$ generally depends on the choice of $\Lambda$ but is independent of the choice of the initial conditions $q\left(t_{0}\right)$ and $\dot{q}\left(t_{0}\right)$.

Proof. See Appendix (8).

In Fig. 3, we illustrate the geometric relation between the reduced model flow on the slow manifold to general trajectories of the full system, as described by Theorem 1 .

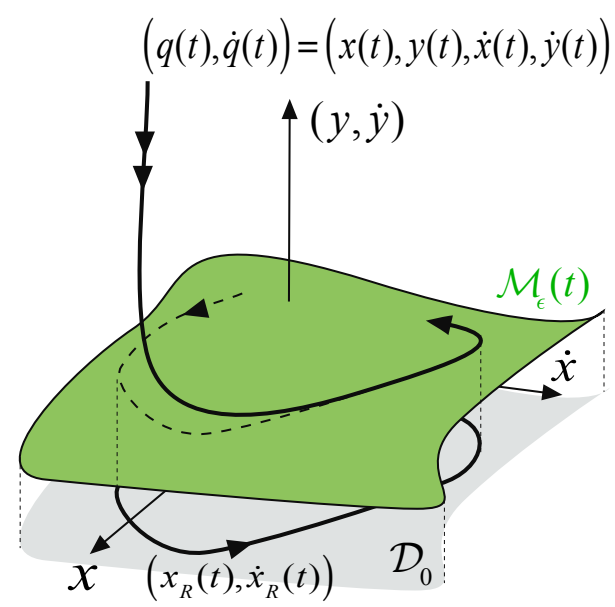

Figure 3: Reduced-order model trajectory $\left(x_{R}(t), \dot{x}_{R}(t)\right)$ as a projection from the slow manifold $\mathcal{M}_{\epsilon}(t)$ to the space of the $(x, \dot{x})$ variables. Other nearby trajectories converge to the slow manifold exponentially fast, and hence their projection on the $(x, \dot{x})$ space synchronizes exponentially with trajectories of the reduced-order model.

Remark 1. If the left-hand side of the reduced-order model $(18)$ has structurally stable features (cf. Guckenheimer and Holmes [15]), then, for $\epsilon>0$ small enough, these features persist smoothly under the addition of the $\mathcal{O}(\epsilon)$ terms of the right-hand side, and hence an explicit computation of these terms is not necessary. For instance, if system (18) has a single attracting fixed point or periodic orbit over the compact domain $\mathcal{D}_{0}$, then wither of these features is robust without the explicit inclusion of the $\mathcal{O}(\epsilon)$ and higher-order terms on its right-hand side. If, however, system (18) is conservative, then the inclusion of $\mathcal{O}(\epsilon)$ terms is necessary to obtain a robust, dissipative reduced-order model. If the $\mathcal{O}(\epsilon)$ terms are also conservative, then explicit evaluation of the $\mathcal{O}\left(\epsilon^{2}\right)$ is required following the expansion scheme used in the proof of Theorem 1 . 
Remark 2. The synchronization expressed by (21) means that both positions and velocities predicted by the reduced-order model (18) are relevant for the observed system dynamics as long the time $t>t_{0}$ is selected from the domain $\mathcal{T}$. This time-domain is unbounded (i.e., $\mathcal{T}=\mathbb{R}$ ) for mechanical systems with explicit periodic and quasiperiodic time dependence. For the case of temporally aperiodic time dependence, the times allowed in 221) are restricted to the finite interval $\mathcal{T}=[a, b]$.

Example 5. [Partially stiff weakly nonlinear system with parametric forcing] We recall that the partially stiff system (14) in Example 4 satisfies assumptions (A1)-(A3) and hence admits an exact, global reduced-order model. The form of the function $G_{0}$ from 15 is

$$
G_{0}(x, \dot{x}, t)=K_{2}^{-1}\left[f_{2}(t)-S_{2}(x, 0)\right] .
$$

The mass matrix $M_{1}$ is independent of $\epsilon$, and hence the equivalent form 119 ) of the reduced-order model applies and gives

$$
M_{1} \ddot{x}+C_{1} \dot{x}+K_{1} x+S_{1}\left(x, K_{2}^{-1}\left[f_{2}(t)-S_{2}(x, 0)\right]\right)=f_{1}(t)+O(\epsilon) .
$$

The leading-order terms in the expressions 20 for the slow manifold are

$$
\begin{aligned}
& y=\epsilon G_{0}(x, \dot{x}, t)+\mathcal{O}\left(\epsilon^{2}\right)=\epsilon K_{2}^{-1}\left[f_{2}(t)-S_{2}(x, 0)\right]+\mathcal{O}\left(\epsilon^{2}\right) \\
& \dot{y}=\epsilon H_{0}(x, \dot{x}, t)+\mathcal{O}\left(\epsilon^{2}\right)=\epsilon K_{2}^{-1}\left[\dot{f}_{2}(t)-\partial_{x} S_{2}(x, 0) \dot{x}\right]+\mathcal{O}\left(\epsilon^{2}\right) .
\end{aligned}
$$

If $f(t)$ is periodic or quasiperiodic in time, then we have the synchronization estimate 21) for all times $t>t_{0}$. Specifically, any $\Lambda>0$ can be selected such that $-\Lambda<0$ is a strict upper bound on the real part of the spectrum of the oscillatory system (16). We note that if we had assumed a non-stiff coupling of the form $S_{1}(x, y)$ in Example 4, then assumptions (A1)-(A4) would still have been satisfied, but the reduced model would simplify to

$$
M_{1} \ddot{x}+C_{1} \dot{x}+K_{1} x+S_{1}(x, 0)=f_{1}(t)+O(\epsilon),
$$

uncoupling completely from the stiff modes at leading order. The convergence estimate (21) would remain valid in this case, too.

\section{The boundary of the domain of model reduction}

In the examples we have discussed so far, the domain $\mathcal{D}_{0}$ could be selected arbitrarily large. Thus, a reduced-order model exists over arbitrarily large $(x, \dot{x}, t)$ values in these problems, as long as $\epsilon$ is kept small enough. In general, however, $\mathcal{D}_{0}$ will have a nonempty boundary $\partial \mathcal{D}_{0}$ over which the reduced-order model (18)-(19) cannot be further extended.

Such non-extendibility of the reduced-order model domain arises from a break-down in the solvability of the algebraic equation (7) for the critical manifold. By the implicit function theorem, this occurs along points satisfying

$$
\operatorname{det}\left[\partial_{\eta} P_{2}\left(x, \dot{x}, G_{0}(x, \dot{x}, t), 0, t ; 0\right)\right]=0, \quad(x, \dot{x}, t) \in \partial \mathcal{D}_{0} .
$$

In the generic case, this determinant becomes zero at points where $\partial_{\eta} P_{2}$ has a single zero eigenvalue, i.e.,

$$
\operatorname{rank}\left[\partial_{\eta} P_{2}\left(x, \dot{x}, G_{0}(x, \dot{x}, t), 0, t ; 0\right)\right]=f-1, \quad(x, \dot{x}, t) \in \partial \mathcal{D}_{0} .
$$

Under further nondegeneracy conditions (see., e..g., Arnold [2]), a fold develops in the critical manifold along $\partial \mathcal{D}_{0}$, i.e., $\mathcal{M}_{0} \equiv \mathcal{M}_{0}^{+}$ceases to be a locally unique graph over the $(x, \dot{x}, t)$ variables. As we pass from $\mathcal{M}_{0}$ to the newly bifurcating critical manifold branch $\mathcal{M}_{0}^{-}$, the matrices $A(x, \dot{x}, t)$ and $B(x, \dot{x}, t)$ vary smoothly in their arguments, given that one manifold branch is smoothly connected to the other one along a fold. Under the nondegeneracy condition (24), precisely one eigenvalue of 
the matrix $B(x, \dot{x}, t)$ will cross zero in the passage from $\mathcal{M}_{0}^{+}$to $\mathcal{M}_{0}^{-}$along the critical manifold. In this case, the graph segment $\eta=G_{0}^{-}(x, \dot{x}, t)$ describing the bifurcating branch $\mathcal{M}_{0}^{-}$(9) violates assumption (A2). As a consequence, the folded slow manifold branch $\mathcal{M}_{\epsilon}^{-}$perturbing from $\mathcal{M}_{0}^{-}$is unstable and hence irrelevant for reduced-order modeling.

In summary, unlike in the setting of the local construction of spectral submanifolds near equilibria (cf. Haller and Ponsioen [17]), a folding invariant manifold arising in SFD is not a technical limitation to overcome when one is in pursuit of a more global reduced-order model. Rather, a fold in the slow manifold over the plane of slow variables signals precisely the limit beyond which no reduced-order model satisfying (R1)-(R2) exists in a given part of the phase space.

Example 6. [Partially stiff weakly nonlinear system with parametric forcing] Consider now the multi-degree-of-freedom mechanical system

$$
\begin{aligned}
M_{1} \ddot{x}+C_{1} \dot{x}+K_{1} x+S_{1}\left(x, \frac{y}{\epsilon}\right) & =f_{1}(t), \\
\epsilon M_{2} \ddot{y}+C_{2} \dot{y}+\frac{1}{\epsilon} K_{2} y+S_{2}\left(x, \frac{y}{\epsilon}\right) & =f_{2}(t),
\end{aligned}
$$

with the same variables, matrices and functions used in Example 4, except that here the coupling function $S_{2}$ also has a stiff dependence on the $y$ variables. We then obtain

$$
\begin{aligned}
& P_{1}(x, \dot{x}, \eta, \dot{y}, t ; \epsilon)=-M_{1}^{-1}\left[C_{1} \dot{x}+K_{1} x+S_{1}(x, \eta)-f_{1}(t)\right], \\
& P_{2}(x, \dot{x}, \eta, \dot{y}, t ; \epsilon)=-M_{2}^{-1}\left[C_{2} \dot{y}+K_{2} \eta+S_{2}(x, \eta)-f_{2}(t)\right],
\end{aligned}
$$

thus assumption (A1) is satisfied again. The condition $(23)$ in this case gives

$$
\operatorname{det}\left[M_{2}^{-1}\left(K_{2}+\partial_{y} S_{2}(x, \eta)\right)\right] \neq 0 .
$$

By the non-singularity of $M_{2}$, this latter condition is equivalent to

$$
\operatorname{det}\left[K_{2}+\partial_{y} S_{2}(x, \eta)\right] \neq 0 .
$$

For instance, when $S_{2}$ has only quadratic terms, then this last condition can always be written as

$$
\operatorname{det}\left[K_{2}+\Pi x+\Phi \eta\right] \neq 0,
$$

where $\Pi$ and $\Phi$ are 3-tensors of appropriate dimensions. Suppose now, for simplicity, that $\Phi \equiv 0$, the master variable $x$ is a scalar $(m=1)$, and $\Pi \in \mathbb{R}^{f \times f}$ is nonsingular. The requirement (26) then becomes

$$
\operatorname{det}\left[\Pi^{-1} K_{2}-(-x) I\right] \neq 0,
$$

which implies that $-x$ cannot be an eigenvalue of $\Pi^{-1} K_{2}$. Consequently, condition (27) fails along the domain boundary

$$
\partial \mathcal{D}_{0}=\left\{(x, \dot{x}, t): \exists j: \quad x=-\lambda_{j}\left(\Pi^{-1} K_{2}\right),\right\},
$$

with $\lambda_{j}\left(\Pi^{-1} K_{2}\right)$ denoting the $j^{\text {th }}$ real eigenvalue of the matrix $\Pi^{-1} K_{2}$.

To illustrate the geometry of the critical manifold in a simple case, we let $s=f=1$ and select the parameters, the coupling and the forcing terms as

$$
K_{2}=4, \quad S_{2}(x, \eta)=x^{2}+4 \eta^{2}, \quad f_{2}(t)=\sin t,
$$

so that the equation $P_{2}(x, \dot{x}, \eta, 0, t ; 0)=0$ takes the form

$$
4 \eta+x^{2}+4 \eta^{2}-\sin t=0 .
$$


This equation is solved by $\eta=x=t=0$ and hence the set $\mathcal{D}_{0}$ is nonempty. The boundary $\partial \mathcal{D}_{0}$, defined by conditions (23)-(24), satisfies

$$
\begin{aligned}
\operatorname{det}\left[\partial_{\eta} P_{2}(x, \dot{x}, \eta, 0, t ; 0)\right] & =\partial_{\eta} P_{2}(x, \dot{x}, \eta, 0, t ; 0)=4+8 \eta=0 \quad \Longleftrightarrow \quad \eta=-\frac{1}{2}, \\
\partial_{\eta} \operatorname{det}\left[\partial_{\eta} P_{2}\left(x, \dot{x},-\frac{1}{2}, 0, t ; 0\right)\right] & =8 \neq 0,
\end{aligned}
$$

where the second condition here is the classic nondegeneracy condition for fold bifurcations in the one-dimensional case (cf. Arnold [2]). Substitution of $\eta=-\frac{1}{2}$ into the equation(28) gives an explicit definition for $\partial \mathcal{D}_{0}$ in the $(x, \dot{x}, t)$ space as

$$
\partial \mathcal{D}_{0}=\left\{(x, \dot{x}, t): x^{2}=1+\sin t\right\} .
$$

A direct solution of equation (28) through the quadratic formula confirms that the zero set

$$
\eta=G_{0}^{ \pm}(x, \dot{x}, t)=\frac{-1 \pm \sqrt{1-\left(x^{2}-\sin t\right)}}{2}, \quad(x, \dot{x}, t) \in \mathcal{D}_{0}=\left\{(x, \dot{x}, t): x^{2}<1+\sin t\right\}
$$

indeed ceases to be a graph and develops a fold singularity along $\partial \mathcal{D}_{0}$. The stability of the two branches of $G_{0}^{ \pm}(x, \dot{x}, t)$ can be determined by calculating (9) along both branches:

$$
\begin{aligned}
& A^{ \pm}(x, \dot{x}, t)=M_{2}^{-1} C_{2}, \\
& B^{ \pm}(x, \dot{x}, t)=-M_{2}^{-1}\left(4+8 G_{0}^{ \pm}(x, \dot{x}, t)\right)=\mp 4 \sqrt{1-\left(x^{2}-\sin t\right)} .
\end{aligned}
$$

Therefore, the critical manifold

$$
\mathcal{M}_{0}^{+}=\left\{(x, \dot{x}, t) \in \mathcal{D}_{0}: \eta=G_{0}^{+}(x, \dot{x}, t)\right\}
$$

satisfies assumption (A1)-(A3) but develops a fold over $\mathcal{D}_{0}$ along the boundary curve $\partial \mathcal{D}_{0}$ defined in (29). The additional branch

$$
\mathcal{M}_{0}^{-}=\left\{(x, \dot{x}, t) \in \mathcal{D}_{0}: \eta=G_{0}^{-}(x, \dot{x}, t)\right\}
$$

emanating from the domain boundary $\partial \mathcal{D}_{0}$ is unstable, as its associated constant-coefficient linear system (cf. assumption (A2)), given by

$$
M_{2} u^{\prime \prime}+C_{2} u^{\prime}-4 \sqrt{1-\left(x^{2}-\sin t\right)} u=0,
$$

is unstable. We show the stable and unstable critical manifolds, as well as the domain boundary $\partial \mathcal{D}_{0}$, in Fig. 4 


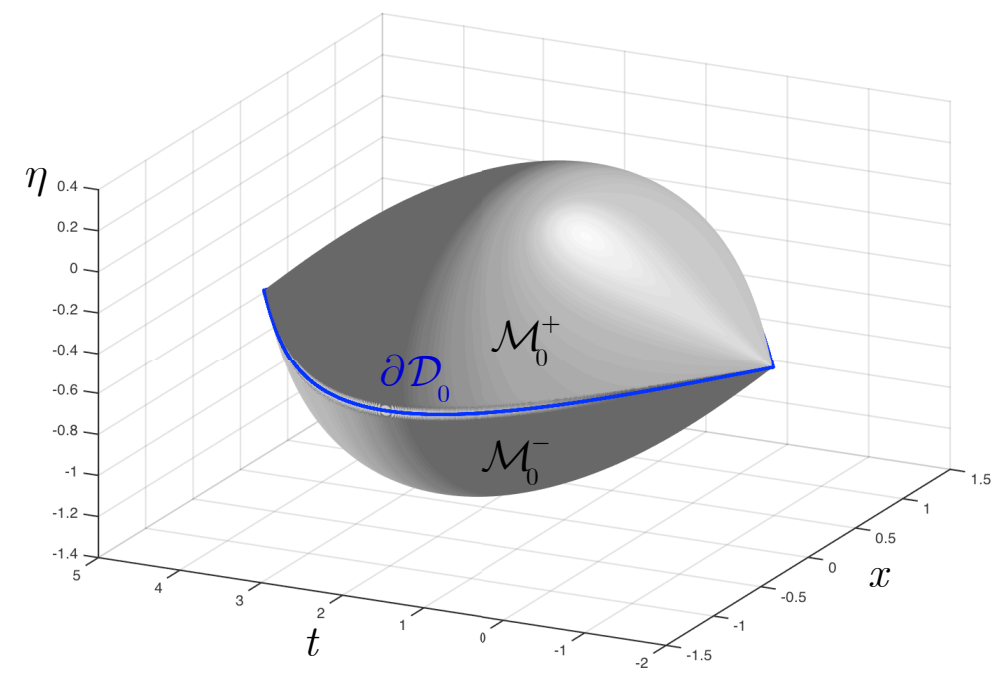

Figure 4: The stable critical manifold branch $\mathcal{M}_{0}^{+}$and the unstable branch $\mathcal{M}_{0}^{-}$for the nonlinear mechanical system (25) with $s=f=1$. Also shown is the domain boundary $\partial \mathcal{D}_{0}$ along which the fold in the critical manifold $\mathcal{M}_{0}$ develops.

\section{Approximate SFD near equilibria: Static condensation and modal derivatives}

Here we show that at least two formal reduction procedures used in structural dynamics, modal condensation and the method of modal derivatives, can be mathematically justified when the conditions (A1)-(A3) of the SFD are satisfied. In this case, these two procedures turn out to provide local first- and second-order approximations, respectively, to a slow manifold $\mathcal{M}_{\epsilon}$ emanating from an equilibrium point of the unforced mechanical system

To show this, we also assume the following:

(A4) Independence of critical manifold of the slow velocities: The relation

$$
\partial_{\dot{x}} Q_{2}(x, \dot{x}, \eta, 0, t ; 0) \equiv 0,
$$

holds, i..e, the function $P_{2}$ has not explicit dependence on the slow velocities $\dot{x}$ for $\epsilon=0$ and $\dot{y}=0$.

We further assume that the domain $\mathcal{D}_{0}$, over which the graph $\eta=G_{0}(x, \dot{x}, t)$ is defined, contains the line $x=0$ of the $(x, \dot{x}, t)$ parameter space, i..e,

(A5) Critical manifold contains an unforced fixed point: We assume

$$
\{(x, \dot{x}, t): x=0, \quad \dot{x}=0\} \subset \mathcal{D}_{0} .
$$

This condition is satisfied, for instance, when (1) is a weakly nonlinear system whose unforced part admits a fixed point at $q=(x, y)=0$. The implications of assumptions (A4)-(A5) for the geometry of the critical manifold are illustrated in Fig. 5.

Static condensation (Geradin and Rixen [14]) is a linear reduction procedure applied to a $q=(x, y)$ partition of the degrees of freedom in system (1) near an equilibrium point. In this reduction method, the inertial terms and velocities are simply ignored in the linearized equation for the $y$ degrees of freedom. The resulting linear algebraic equation is solved for $y$, and the result is substituted for $y$ in the $x$ equations, yielding a single second-order differential equation in the $x$ variables. 


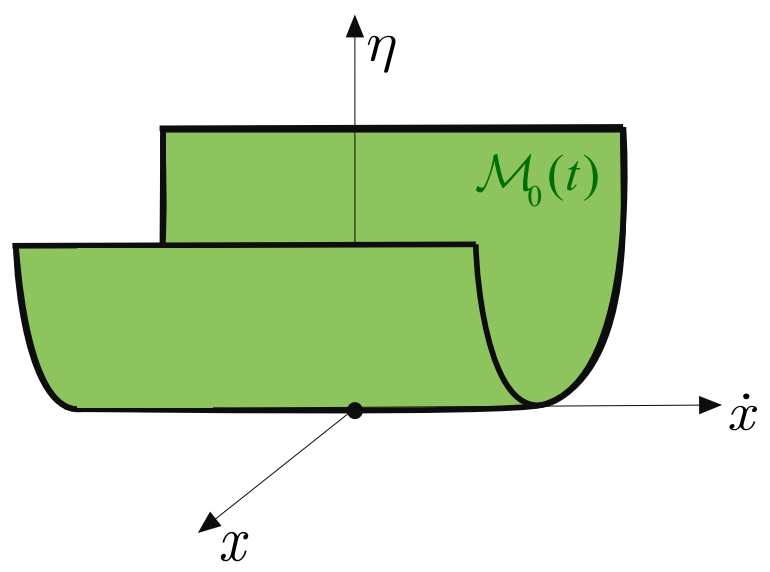

Figure 5: The geometry of the critical manifold $\mathcal{M}_{0}(t)$ under assumptions (A3)-(A4) at an arbitrary time $t$.

The method of modal derivatives (Idelsohn and Cardona [18, Rutzmoser et al. 28], Wu and Tiso [33]) considers a similar $q=(x, y)$ partition of coordinates near an unforced equilibrium and seeks a quadratic invariant manifold tangent to an eigenspace of the linearized system. The main assumption is that along this quadratic manifold, the $y$ coordinates can be written as purely quadratic functions of the $x$ coordinates, with the coefficients of this quadratic forms collected in an appropriate modal derivative tensor.

The above two reduction methods can be justified in our present setting as follows:

Proposition 1. Under assumptions (A1)-(A5):

(i) The expressions derived for the slow manifold $\mathcal{M}_{\epsilon}(t)$ in 20 satisfy

$$
G_{0}(x, \dot{x}, t)=\Gamma(t)+\Phi(t) x+(\Theta(t) x) x+\mathcal{O}\left(|x|^{3}\right),
$$

where the function $\Gamma(t)$ is the solution of the equation $P_{2}(0, \Gamma(t), 0, t ; 0)=0$, and the two-tensor $\Phi(t)$ and the three-tensor $\Theta(t)$ satisfy

$$
\begin{aligned}
& \Phi(t)=-\left.\left[\partial_{\eta} P_{2}\right]^{-1} \partial_{x} P_{2}\right|_{x=0, \eta=\Gamma(t), \dot{y}=0, \epsilon=0}, \\
& \Theta(t)=-\left.\frac{1}{2}\left[\partial_{\eta} P_{2}\right]^{-1}\left[\partial_{x x}^{2} P_{2}+\left(2 \partial_{x \eta}^{2} P_{2}+\partial_{\eta \eta}^{2} P_{2} \Phi(t)\right) \Phi(t)\right]\right|_{x=0, \eta=\Gamma(t), \dot{y}=0, \epsilon=0} .
\end{aligned}
$$

(ii) Assume that $P_{2}(x, \dot{x}, \eta, 0, t ; 0)$ has no explicit time dependence and $(x, y)$ are modal coordinates for the linearized system at $(x, y)=0$, i.e.,

$$
\partial_{t} P_{2}(x, \dot{x}, \eta, 0, t ; 0) \equiv 0, \quad \partial_{x} P_{2}(0,0,0,0, t ; 0)=0, \quad \partial_{\eta} P_{1}(0,0,0,0, t ; 0)=0 .
$$

We then obtain

$$
\Gamma=0, \quad \Phi=0, \quad \Theta=-\frac{1}{2}\left[\partial_{\eta} P_{2}(0,0,0,0, t ; 0)\right]^{-1} \partial_{x x}^{2} P_{2}(0,0,0,0, t ; 0) .
$$

(iii) Under the conditions of statement (ii), a linear-in-x and zeroth-order-in- $\epsilon$ approximation to $\mathcal{M}_{\epsilon}(t)$ yields the modal-condensation-based reduced model

$$
\ddot{x}-P_{1}(x, \dot{x}, 0,0, t ; 0)+\mathcal{O}\left(\epsilon,|x|^{3}\right)=0
$$

for the dynamics on $\mathcal{M}_{\epsilon}(t)$ 
(iv) Under the conditions of statement (ii), a quadratic-in-x and zeroth-order-in- $\epsilon$ approximation to $\mathcal{M}_{\epsilon}(t)$ yields the modal-derivatives-based reduced-order model

$$
\ddot{x}-P_{1}(x, \dot{x},(\Theta(t) x) x, 0, t ; 0)+\mathcal{O}\left(\epsilon,|x|^{4}\right)=0
$$

for the dynamics on $\mathcal{M}_{\epsilon}(t)$, with $\Theta(t)$ generally referred to as the modal derivative tensor.

Proof. See Appendix 9

Remark 3. Combining statement (iii) of Theorem 1 with Proposition 1 gives that if $M_{1}(x, \eta, \epsilon)$ is smooth in $\epsilon$ at $\epsilon=0$, then the static-condensation-based model 35 is equivalent to

$$
M_{1}(x, 0 ; 0) \ddot{x}-Q_{1}(x, \dot{x}, 0,0, t ; 0)+\mathcal{O}\left(\epsilon,|x|^{3}\right)=0,
$$

and the modal-derivatives-based reduced model 36 is equivalent to

$$
M_{1}(x,(\Theta x) x ; 0) \ddot{x}-Q_{1}(x, \dot{x},(\Theta x) x, 0, t ; 0)+\mathcal{O}\left(\epsilon,|x|^{4}\right)=0 .
$$

Remark 4. The unevaluated higher-order $\mathcal{O}\left(|x|^{3}\right)$ and $\mathcal{O}\left(|x|^{4}\right)$ terms in eqs. (37) and (38) generally do not remain uniformly small over the full model-reduction domain $\mathcal{D}_{0}$. Rather, one can only use the leading-order model terms in these equations reliably as long as the slow coordinates are rescaled as $x=\sqrt[3]{\epsilon} \xi$ and $x=\sqrt[4]{\epsilon} \xi$, respectively. In that case, (37) and (38) can be re-written as

$$
\begin{aligned}
M_{1}(\xi, 0 ; 0) \ddot{\xi}-Q_{1}(\xi, \dot{\xi}, 0,0, t ; 0)+\mathcal{O}(\epsilon) & =0, \\
M_{1}(\xi,(\Theta \xi) \xi ; 0) \ddot{\xi}-Q_{1}(\xi, \dot{\xi},(\Theta \xi) \xi, 0, t ; 0)+\mathcal{O}(\epsilon) & =0,
\end{aligned}
$$

respectively. One can then arguably focus on the $\epsilon$-independent leading order terms for $\epsilon>0$ small enough. The static-condensation- and model-derivative-based reductions are, therefore, justified in order $\mathcal{O}(\sqrt[3]{\epsilon})$ and $\mathcal{O}(\sqrt[4]{\epsilon})$ neighborhoods of the $x=0$ equilibrium, respectively, provided that the assumptions of Proposition 1 are satisfied.

Example 7. [Localized reduced-order model for a stiff, weakly nonlinear system with parametric forcing] We reconsider now the multi-degree-of-freedom mechanical system (14) and assume that

$$
f_{2}(t) \equiv 0,
$$

i.e., that the external forcing on the stiff degrees of freedom vanishes. Using the results from Example 6. we have

$$
\begin{aligned}
P_{1}(x, \dot{x}, \eta, \dot{y}, t ; \epsilon) & =-M_{1}^{-1}\left[C_{1} \dot{x}+K_{1} x+S_{1}(x, \eta)-f_{1}(t)\right] \\
P_{2}(x, \dot{x}, \eta, \dot{y} ; \epsilon) & =-M_{2}^{-1}\left[C_{2} \dot{y}+K_{2} \eta+S_{2}(x, \eta)\right] .
\end{aligned}
$$

As already discussed in Example 6, conditions (A1)-(A3) are satisfied and hence Theorem 1 guarantees a slow manifold and determines its reduced dynamics. Assumption (30) is clearly satisfied, as $P_{2}$ does not depend on $\dot{x}$. Assumption (31) also holds, as one sees from the expression for $G_{0}$ in 22 . Since $P_{2}$ has no explicit time dependence, the static condensation and modal derivative formulas in 83) apply and take the specific form

$$
\Gamma \equiv 0, \quad \Phi \equiv 0, \quad \Theta=-\left[-M_{2}^{-1} K_{2}\right]^{-1}\left[-M_{2}^{-1} \partial_{x x}^{2} S_{2}(0,0)\right]=-K_{2}^{-1} \partial_{x x}^{2} S_{2}(0,0) .
$$

Therefore, in a neighborhood of the origin, the reduced-order formulation (39) applies and statement (iii) of Proposition 1 justifies the static-condensation-based reduced model

$$
M_{1} \ddot{\xi}+C_{1} \dot{\xi}+K_{1} \xi+S_{1}(x, 0)-f_{1}(t)+\mathcal{O}(\epsilon)=0
$$


as a leading-order reduced model for the dynamics on $\mathcal{M}_{\epsilon}(t)$ in an order $\mathcal{O}(\sqrt[3]{\epsilon})$ neighborhood of the unforced equilibrium $x=0$. Similarly, statement (iv) of Proposition (1) justifies the modalderivatives-based reduced-order model

$$
M_{1} \ddot{\xi}+C_{1} \dot{\xi}+K_{1} \xi+S_{1}\left(x,-\left[K_{2}^{-1} \partial_{x x}^{2} S_{2}(0,0) x\right] x\right)-f_{1}(t)+\mathcal{O}(\epsilon)=0
$$

in an order $\mathcal{O}(\sqrt[4]{\epsilon})$ neighborhood of the unforced equilibrium $x=0$.

The above example illustrates how Proposition 1 puts static condensation and modal derivatives in a rigorous context under appropriate assumptions. We now also illustrate, however, that these two intuitive reduction methods give incorrect results when the assumptions of Proposition 1 are not satisfied.

Example 8. [Failure of static modal condensation and model-derivative-based reduction] Consider a two-degree-of-freedom nonlinear, coupled oscillator system with amplitude-dependent damping in the first mode, given by the equations

$$
\begin{aligned}
\ddot{x}+\left(c_{1}+\mu_{1} x^{2}\right) \dot{x}+k_{1} x+a x y+b x^{3} & =0, & x \in \mathbb{R}, \\
\ddot{y}+c_{2} \dot{y}+k_{2} y+c x^{2} & =0, & y \in \mathbb{R} .
\end{aligned}
$$

Note that the linearized system at the $(x, y)=(0,0)$ equilibrium is in modal coordinates. For $c_{2}>c_{1}$, we obtain slower linear amplitude decay in the two-dimensional modal subspace of the $x$ variable than in the modal subspace of the $y$ variable. This suggests a reduction to a model involving only the slower $x$ variables. The argument used in Example 1 . however, shows that (44) violates assumption (A3) and hence Proposition 1 does not apply. The static condensation procedure nevertheless gives the formal reduced-order model

$$
\ddot{x}+\left(c_{1}+\mu_{1} x^{2}\right) \dot{x}+k_{1} x+b x^{3}=0,
$$

and the method of modal-derivates formally gives the formal reduced model

$$
\ddot{x}+\left(c_{1}+\mu_{1} x^{2}\right) \dot{x}+k_{1} x+\left(b-\frac{a c}{k_{2}}\right) x^{3}=0,
$$

modifying 45 at cubic order only. While a global slow manifold is not guaranteed to exist in this example, a unique, two-dimensional analytic invariant manifold tangent to the subspace of the $x$ variables at the origin does exist (cf. Haller and Ponsioen [17]). This spectral submanifold (SSM) offers a mathematically rigorous process for model reduction in system (44), providing an exact reduced flow to which (45) and (46) can be compared. As we show in Appendix 10, the reduced model on the slow SSM is of the form

$$
\begin{aligned}
& \ddot{x}+\left[c_{1}+\left(\mu_{1}-\frac{2 a c\left(4 c_{1} k_{1}+k_{2}\left(c_{1}-c_{2}\right)+2 c_{1} c_{2}^{2}-6 c_{1}^{2} c_{2}+4 c_{1}^{3}\right)}{D}\right) x^{2}\right] \dot{x} \\
& +\left[k_{1}-\frac{2 a c\left(2 c_{1}^{2}-3 c_{1} c_{2}+c_{2}^{2}+4 k_{1}-k_{2}\right)}{D} \dot{x}^{2}\right] x \\
& +\left[b-\frac{a c\left(4 c_{1}^{4}-6 c_{1}^{3} c_{2}+2 c_{1}^{2} c_{2}^{2}+5 c_{1}^{2} k_{2}-c_{1} c_{2}\left(2 k_{1}+3 k_{2}\right)+2 c_{2}^{2} k_{1}+8 k_{1}^{2}-6 k_{1} k_{2}+k_{2}^{2}\right)}{D}\right] x^{3} \\
& +\mathcal{O}(4)=0,
\end{aligned}
$$

where

$$
D=\left(c_{1}^{2}-c_{1} c_{2}+k_{2}\right)\left(4 c_{1}^{2} k_{2}-8 c_{1} c_{2} k_{1}-2 c_{1} c_{2} k_{2}+4 c_{2}^{2} k_{1}+16 k_{1}^{2}-8 k_{1} k_{2}+k_{2}^{2}\right) .
$$


A comparison of the exact reduced model 47 with the statically condensed version 45 and with the modal-derivatives-based version (46) shows that the latter two heuristic reduction methods miss most terms already in the leading-order (cubic) nonlinearities. Depending on the specific value of the parameters, the missing terms can significantly impact the nature of the reduced dynamics and hence cannot be omitted. We note that in the slow-fast limit expressed by the scaling

$$
c_{2} \rightarrow c_{2} / \epsilon, \quad k_{2} \rightarrow k_{2} / \epsilon^{2},
$$

system (44) satisfies the assumptions of Proposition 1 and hence the approximation to the slow SSM should coincide with the approximation to the global slow manifold $\mathcal{M}_{\epsilon}$ in this case. Indeed, in this scaling, formulas 89 for the constants $\alpha, \beta$ and $\gamma$ in Appendix 10 simplify to

$$
\alpha=-\frac{c}{k_{2}} \epsilon^{2}+\mathcal{O}\left(\epsilon^{3}\right), \quad \beta=\mathcal{O}\left(\epsilon^{3}\right), \quad \gamma=\mathcal{O}\left(\epsilon^{3}\right),
$$

and hence the exact reduced model (47) simplifies to

$$
\ddot{x}+\left(c_{1}+\mu_{1} x^{2}\right) \dot{x}+k_{1} x+\left(b-\frac{a c}{k_{2} / \epsilon^{2}}\right) x^{3}+\mathcal{O}\left(\epsilon^{3}\right)=0,
$$

coinciding with the modal-derivatives-based reduced-order model (46). This agreement, however, only holds in the slow-fast setting (49).

Even in the conservative limit, when the SSM is replaced by a unique, analytic Lyapunovsubcenter manifold (Kelley [21]), we obtain a conservative limit of the exact reduced-order model (47) in the form

$$
\ddot{x}+\left[k_{1}-\frac{2 a c}{k_{2}\left(4 k_{1}-k_{2}\right)} \dot{x}^{2}\right] x+\left[b-\frac{a c\left(2 k_{1}-k_{2}\right)}{k_{2}\left(4 k_{1}-k_{2}\right)}\right] x^{3}+\mathcal{O}\left(x^{4}\right)=0,
$$

filled with nonlinear normal modes (periodic orbits). At the same time, the conservative limit of the static condensation procedure gives

$$
\ddot{x}+k_{1} x+b x^{3}=0,
$$

while the modal derivatives-based reduction 46 gives

$$
\ddot{x}+k_{1} x+\left(b-\frac{a c}{k_{2}}\right) x^{3}+\mathcal{O}\left(x^{4}\right)=0 .
$$

Comparing (51) and (53) shows that the method of modal derivatives gives an incorrect reducedorder model up to cubic order, unless we have either $a=0$ or $c=0$. As shown in Fig. 6, the error between the actual reduced flow (51) and (53) grows unbounded in the vicinity of the $2: 1$ resonance (represented by $k_{2}=4 k_{1}$ ) between the two natural frequencies of the undamped limit of system (44). In the limit of an exact $2: 1$ resonance, no invariant manifold tangent to the $x$-subspace exists, even though the modal derivative approach still suggests the existence a bounded reduced flow on such a manifold. 

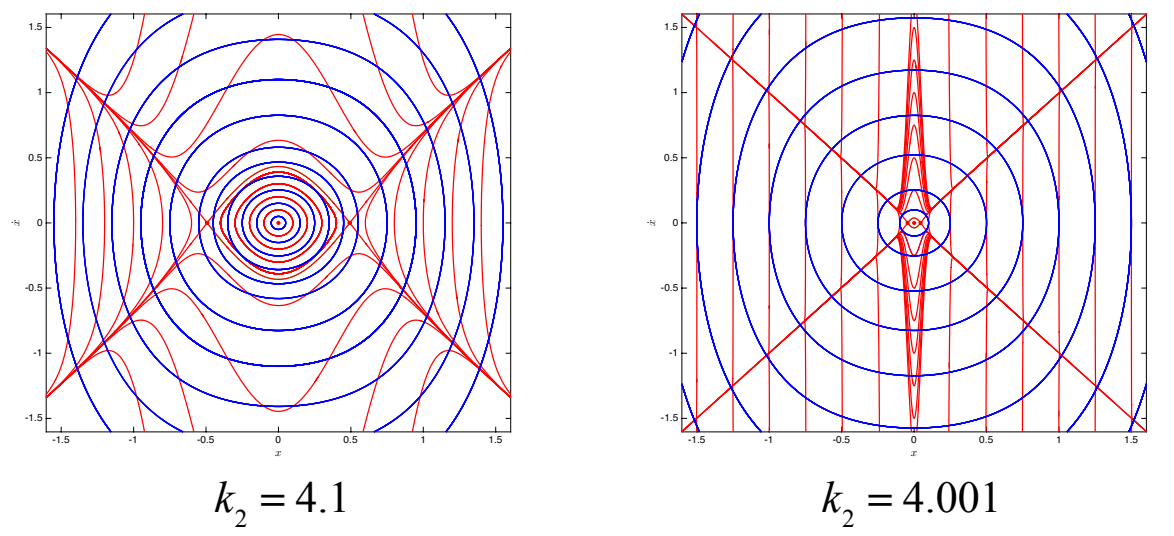

Figure 6: Trajectories of the cubic modal-derivatives-based reduction (53) (blue) and those of the exact cubic reduction (51) (red) to the unique, 2D analytic invariant manifold over the $(x, \dot{x})$ variables. The remaining parameters are set as $k_{1}=a=b=c=1$.

\section{A detailed example: Three-degree-of-freedom system with a pendulum damper}

We consider a the system depicted in Fig. 7, with a mass $M$ hanging on a vertical spring of unstretched length $L$ and linear viscous damping $C_{h}$. The spring is hardening, with linear stiffness coefficient $K_{h}$ and cubic stiffness coefficient $\Gamma_{h}>0$. The mass is subject to downward external periodic forcing of the form $f_{h}(t)=f_{h_{0}} \sin \omega_{1} t$, as well as to gravity whose constant is $g$. The downward position of the mass from the unstretched spring position is measured by the coordinate $h$. The horizontal spring with linear stiffness coefficient $K_{d}$ and natural length $D$ is fixed to the surroundings, thereby introducing geometric nonlinearities. Added in this direction is a viscous damper with damping coefficient $C_{d}$ and an external periodic force $f_{d}(t)=f_{d_{0}} \sin \omega_{1} t$, both acting in the horizontal direction.

As indicated in Fig. 7) a pendulum of mass $m$ and length $l$ is attached to the mass $M$. The angle of the pendulum from the vertical is denoted by $\gamma$. The pendulum is also subject to angular viscous damping with coefficient $c_{p}$, and to an external periodic force $f_{p}(t)=f_{p_{0}} \sin \omega_{2} t$ acting on $m$ in a direction normal to the pendulum . 


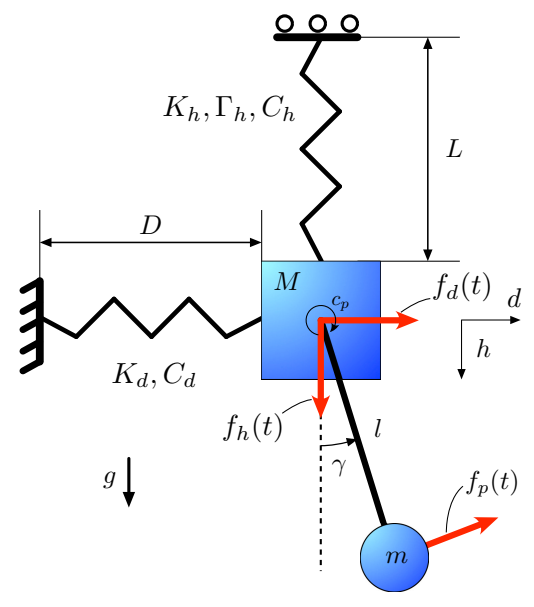

Figure 7: Three degree-of-freedom coupled pendulum

The equations of motion for this system are

$$
\begin{aligned}
m l^{2} \ddot{\gamma}-m l \sin \gamma \ddot{h}+m l \cos \gamma \ddot{d}+c_{p} \dot{\gamma}+m g l \sin \gamma & =f_{p}(t) l, \\
(M+m) \ddot{h}-m l \sin \gamma \ddot{\gamma}-m l \cos \gamma \dot{\gamma}^{2}+C_{h} \dot{h}+K_{h} h+K_{d} Q(d, h) h+\Gamma_{h} h^{3} & =(M+m) g+f_{h}(t)-f_{p}(t) \sin \gamma, \\
(M+m) \ddot{d}+m l \cos \gamma \ddot{\gamma}-m l \sin \gamma \dot{\gamma}^{2}+C_{d} \dot{d}+K_{d}(D+d) Q(d, h) & =f_{d}(t)+f_{p}(t) \cos \gamma,
\end{aligned}
$$

with the geometric nonlinear term $Q(d, h)$

$$
Q(d, h)=\left(1-\frac{D}{\sqrt{(D+d)^{2}+h^{2}}}\right) .
$$

The linearized oscillation frequencies of the uncoupled springs and of the pendulum are

$$
\omega_{h}=\sqrt{\frac{K_{h}}{M}}, \quad \omega_{d}=\sqrt{\frac{K_{d}}{M}}, \quad \omega_{p}=\sqrt{\frac{g}{l}},
$$

respectively. With the help of these frequencies, we non-dimensionalize the $h$ and $d$ coordinates, the time $t$, and all system parameters by letting

$$
\begin{gathered}
\tilde{h}=\frac{h}{L}, \quad \tilde{d}=\frac{d}{D}, \quad \tilde{t}=\omega_{p} t, \\
\Delta=\frac{l}{L}, \quad \rho=\frac{D}{L}, \quad \beta=\frac{m}{M}, \quad F_{h}(t)=\frac{f_{h}(t)}{M g}, \quad F_{p}(t)=\frac{f_{p}(t)}{M g}, \quad F_{d}(t)=\frac{f_{d}(t)}{M g}, \quad G_{p}(t)=\frac{f_{p}(t)}{m g}, \\
\pi_{h}=\frac{C_{h}}{\omega_{p} M}, \quad \pi_{d}=\frac{C_{d}}{\omega_{p} M}, \quad \pi_{p}=\frac{c_{p}}{\omega_{p} m L^{2}}, \quad q_{h}=\frac{\omega_{h}^{2}}{\omega_{p}^{2}}, \quad q_{d}=\frac{\omega_{d}^{2}}{\omega_{p}^{2}}, \quad a_{h}=\frac{\Gamma_{h} L^{2}}{M \omega_{p}^{2}},
\end{gathered}
$$

which leads to the following definition for the scaled version of $Q(d, h)$

$$
\tilde{Q}(\tilde{d}, \tilde{h})=\left(1-\frac{\rho}{\sqrt{\rho^{2}(1+\tilde{d})^{2}+\tilde{h}^{2}}}\right) .
$$


Denoting differentiation with respect to the new time $\tilde{t}$ still by a dot, then dropping all the tildes, we obtain the non-dimensionalized equations of motions

$$
\begin{aligned}
\Delta^{2} \ddot{\gamma}-\Delta \sin \gamma \ddot{h}+\rho \Delta \cos \gamma \ddot{d}+\pi_{p} \dot{\gamma}+\Delta^{2} \sin \gamma & =\Delta^{2} G_{p}(t), \\
(1+\beta) \ddot{h}-\beta \Delta \sin \gamma \ddot{\gamma}-\beta \Delta \cos \gamma \dot{\gamma}^{2}+\pi_{h} \dot{h}+q_{h} h+q_{d} h Q(d, h)+a_{h} h^{3} & =(1+\beta) \Delta+F_{h}(t) \Delta-F_{p}(t) \Delta \sin \gamma, \\
(1+\beta) \ddot{d}+\beta \frac{\Delta}{\rho} \cos \gamma \ddot{\gamma}-\beta \frac{\Delta}{\rho} \sin \gamma \dot{\gamma}^{2}+\pi_{d} \dot{d}+q_{d}(1+d) Q(d, h) & =F_{d}(t) \frac{\Delta}{\rho}+F_{p}(t) \frac{\Delta}{\rho} \cos \gamma .
\end{aligned}
$$

\subsection{Two soft degrees of freedom}

We are interested in applying the SFD procedure to system (56) to obtain an exact reduced-order model for the dynamics. First, we assume that $h$ is a stiff degree of freedom and $(\gamma, d)$ represent soft degrees of freedom. In that case, using the notation from system (1), we can write the mass matrix $M(q, t ; \epsilon)$ and the forcing term $F(q, \dot{q}, t ; \epsilon)$ as

$$
\begin{aligned}
M(q, t ; \epsilon)= & \left(\begin{array}{ccc}
\Delta^{2} & \rho \Delta \cos x_{\gamma} & -\Delta \sin x_{\gamma} \\
\beta \frac{\Delta}{\rho} \cos x_{\gamma} & 1+\beta & 0 \\
-\beta \Delta \sin x_{\gamma} & 0 & 1+\beta
\end{array}\right), \\
F(q, \dot{q}, t ; \epsilon)= & \left(\begin{array}{l}
-\pi_{p} \dot{x}_{\gamma}-\Delta^{2} \sin x_{\gamma}+\Delta^{2} G_{p}(t) \\
\beta \frac{\Delta}{\rho} \sin x_{\gamma} \dot{x}_{\gamma}^{2}-\pi_{d} \dot{x}_{d}-q_{d}\left(1+x_{d}\right) Q\left(x_{d}, \frac{y}{\epsilon}\right)+F_{d}(t) \frac{\Delta}{\rho}+F_{p}(t) \frac{\Delta}{\rho} \cos x_{\gamma} \\
\beta \Delta \cos x_{\gamma} \dot{x}_{\gamma}^{2}-\pi_{h} \dot{y}_{h}-q_{h} \epsilon \frac{y}{\epsilon}-q_{d} \epsilon \frac{y}{\epsilon} Q\left(x_{d}, \frac{y}{\epsilon}\right)-a_{h} \epsilon^{3}\left(\frac{y}{\epsilon}\right)^{3} \\
+(1+\beta) \Delta+F_{h}(t) \Delta-F_{p}(t) \Delta \sin x_{\gamma}
\end{array}\right) .
\end{aligned}
$$

Here we have introduced the coordinates $(x, y)$ by letting

$$
x_{\gamma}=\gamma, \quad x_{d}=d, \quad y=h .
$$


The modified mass matrices $M_{i}$ and the forcing terms $Q_{i}$ defined in (5) take the specific form

$$
\begin{aligned}
& M_{1}=M_{11}-M_{12} M_{22}^{-1} M_{21}=\left(\begin{array}{cc}
\frac{\Delta^{2}}{1+\beta}\left(1+\beta \cos ^{2} x_{\gamma}\right) & \rho \Delta \cos x_{\gamma} \\
\beta \frac{\Delta}{\rho} \cos x_{\gamma} & 1+\beta
\end{array}\right), \\
& M_{2}=M_{22}-M_{21} M_{11}^{-1} M_{12}=\frac{1+\beta}{1+\beta \sin ^{2} x_{\gamma}}, \\
& Q_{1}=F_{1}-M_{12} M_{22}^{-1} F_{2}=\left[\begin{array}{l}
q_{1} \\
q_{2}
\end{array}\right] \\
& q_{1}=-\pi_{p} \dot{x}_{\gamma}-\Delta^{2} \sin x_{\gamma}+\Delta^{2} G_{p}(t)+\frac{\Delta \sin x_{\gamma}}{1+\beta}\left[\beta \Delta \cos x_{\gamma} \dot{x}_{\gamma}^{2}-\pi_{h} \dot{y}-q_{h} \epsilon \frac{y}{\epsilon}\right. \\
& \left.-q_{d} \epsilon \frac{y}{\epsilon} Q\left(x_{d}, \frac{y}{\epsilon}\right)-a_{h} \epsilon^{3}\left(\frac{y}{\epsilon}\right)^{3}+(1+\beta) \Delta+F_{h}(t) \Delta-F_{p}(t) \Delta \sin x_{\gamma}\right] \\
& q_{2}=\beta \frac{\Delta}{\rho} \sin x_{\gamma} \dot{x}_{\gamma}^{2}-\pi_{d} \dot{x}_{d}-q_{d}\left(1+x_{d}\right) Q\left(x_{d}, \frac{y}{\epsilon}\right)+F_{d}(t) \frac{\Delta}{\rho}+F_{p}(t) \frac{\Delta}{\rho} \cos x_{\gamma} \\
& Q_{2}=F_{2}-M_{21} M_{11}^{-1} F_{1} \\
& =\beta \Delta \cos x_{\gamma} \dot{x}_{\gamma}^{2}-\pi_{h} \dot{y}-q_{h} \epsilon \frac{y}{\epsilon}-q_{d} \epsilon \frac{y}{\epsilon} Q\left(x_{d}, \frac{y}{\epsilon}\right)-a_{h} \epsilon^{3}\left(\frac{y}{\epsilon}\right)^{3}+(1+\beta) \Delta+F_{h}(t) \Delta-F_{p}(t) \Delta \sin x_{\gamma} \\
& +\frac{(1+\beta) \beta \sin x_{\gamma}}{\Delta\left(1+\beta \sin ^{2} x_{\gamma}\right)}\left[-\pi_{p} \dot{x}_{\gamma}-\Delta^{2} \sin x_{\gamma}+\Delta^{2} G_{p}(t)\right] \\
& -\frac{\beta \rho \sin x_{\gamma} \cos x_{\gamma}}{1+\beta \sin ^{2} x_{\gamma}}\left[\beta \frac{\Delta}{\rho} \sin x_{\gamma} \dot{x}_{\gamma}^{2}-\pi_{d} \dot{x}_{d}-q_{d}\left(1+x_{d}\right) Q\left(x_{d}, \frac{y}{\epsilon}\right)+F_{d}(t) \frac{\Delta}{\rho}+F_{p}(t) \frac{\Delta}{\rho} \cos x_{\gamma}\right] .
\end{aligned}
$$

These give the following expression for the function $P_{1}$

$$
\begin{gathered}
P_{1}(x, v, \eta, w, t ; \epsilon)=M_{1}^{-1}\left[\begin{array}{l}
q_{1 s} \\
q_{2 s}
\end{array}\right], \\
q_{1 s}=-\pi_{p} v_{\gamma}-\Delta^{2} \sin x_{\gamma}+\Delta^{2} G_{p}(t)+\frac{\Delta \sin x_{\gamma}}{1+\beta}\left[\beta \Delta \cos x_{\gamma} v_{\gamma}^{2}-\pi_{h} w_{h}-q_{h} \epsilon \eta\right. \\
\left.-q_{d} \epsilon \eta Q\left(x_{d}, \eta\right)-a_{h} \epsilon^{3} \eta^{3}+(1+\beta) \Delta+F_{h}(t) \Delta-F_{p}(t) \Delta \sin x_{\gamma}\right], \\
q_{2 s}=\beta \frac{\Delta}{\rho} \sin x_{\gamma} v_{\gamma}^{2}-\pi_{d} v_{d}-q_{d}\left(1+x_{d}\right) Q\left(x_{d}, \eta\right)+F_{d}(t) \frac{\Delta}{\rho}+F_{p}(t) \frac{\Delta}{\rho} \cos x_{\gamma},
\end{gathered}
$$

with the inverse of $M_{1}$ given by

$$
M_{1}^{-1}=\frac{1}{\Delta^{2}}\left[\begin{array}{cc}
1+\beta & -\rho \Delta \cos x_{\gamma} \\
-\beta \frac{\Delta}{\rho} \cos x_{\gamma} & \frac{\Delta^{2}}{1+\beta}\left(1+\beta \cos ^{2} x_{\gamma}\right)
\end{array}\right] .
$$

The function $P_{2}$ takes the specific form

$$
\begin{aligned}
P_{2}(x, v, \eta, w, t ; \epsilon)=\epsilon & \left(\frac{1+\beta \sin ^{2} x_{\gamma}}{1+\beta}\right)\left(\beta \Delta \cos x_{\gamma} v_{\gamma}^{2}-\pi_{h} w_{h}-q_{h} \epsilon \eta\right. \\
& -q_{d} \epsilon \eta Q\left(x_{d}, \eta\right)-a_{h} \epsilon^{3} \eta^{3}+(1+\beta) \Delta+F_{h}(t) \Delta-F_{p}(t) \Delta \sin x_{\gamma} \\
& +\frac{(1+\beta) \beta \sin x_{\gamma}}{\Delta\left(1+\beta \sin ^{2} x_{\gamma}\right)}\left[-\pi_{p} v_{\gamma}-\Delta^{2} \sin x_{\gamma}+\Delta^{2} G_{p}(t)\right] \\
& \left.-\frac{\beta \rho \sin x_{\gamma} \cos x_{\gamma}}{1+\beta \sin ^{2} x_{\gamma}}\left[\beta \frac{\Delta}{\rho} \sin x_{\gamma} v_{\gamma}^{2}-\pi_{d} v_{d}-q_{d}\left(1+x_{d}\right) Q\left(x_{d}, \eta\right)+F_{d}(t) \frac{\Delta}{\rho}+F_{p}(t) \frac{\Delta}{\rho} \cos x_{\gamma}\right]\right) .
\end{aligned}
$$


We observe that $\lim _{\epsilon \rightarrow 0} P_{2}(x, v, \eta, w, t ; \epsilon) \equiv 0$, and therefore assumptions (A1)-(A3) are not satisfied without further assumptions on the parameters that ensure the stiff-soft partition of the coordinates. To this end we express the stiffness of the $y$ degree of freedom by letting

$$
\begin{gathered}
\Delta=\frac{l}{L}=\frac{\delta}{\epsilon}, \quad \rho=\frac{D}{L}=\frac{\phi}{\epsilon}, \quad q_{d}=\frac{\omega_{d}^{2}}{\omega_{p}^{2}}=\Omega_{d}^{2}, \quad q_{h}=\frac{\omega_{h}^{2}}{\omega_{p}^{2}}=\frac{\Omega_{h}^{2}}{\epsilon^{2}}, \quad a_{h}=\frac{\alpha_{h}}{\epsilon^{4}}, \\
\pi_{h}=\frac{C_{h}}{\omega_{p} M}=\frac{\mu_{h}}{\epsilon}, \quad \pi_{d}=\frac{C_{d}}{\omega_{p} M}=\mu_{d}, \quad \pi_{p}=\frac{c_{p}}{\omega_{p} m L^{2}}=\frac{c_{p}}{\omega_{p} m\left(\frac{\epsilon}{\delta} l\right)^{2}}=\frac{c_{p} \delta^{2}}{\omega_{p} m \epsilon^{2} l^{2}}=\frac{\mu_{p}}{\epsilon^{2}} .
\end{gathered}
$$

In this parameter range, assumptions (A1)-(A3) are satisfied, as we show in Appendix 11 . The reduced model arising from these calculations is of the form

$$
\begin{aligned}
\ddot{\gamma} & =\frac{\omega_{p}^{2}(M+m)}{M+m \sin ^{2} \gamma}\left(-\frac{c_{p}}{\omega_{p}^{2} m l^{2}} \dot{\gamma}-\sin \gamma+\frac{f_{p}(t)}{m g}\right) \\
- & \frac{\omega_{p}^{2} M \cos \gamma}{M+m \sin ^{2} \gamma}\left(\frac{m}{M \omega_{p}^{2}} \sin \gamma \dot{\gamma}^{2}-\frac{C_{d}}{\omega_{p}^{2} M l} \dot{d}-\frac{K_{d}}{M g} d+\frac{f_{d}(t)}{M g}+\frac{f_{p}(t)}{M g} \cos \gamma\right)+\mathcal{O}(\epsilon), \\
\ddot{d} & =\frac{\omega_{p}^{2} D M}{M+m \sin ^{2} \gamma}\left(\frac{m l}{M D \omega_{p}^{2}} \sin \gamma \dot{\gamma}^{2}-\frac{C_{d}}{M D \omega_{P}^{2}} \dot{d}-\frac{K_{d} l}{M g D} d+\frac{f_{d}(t) l}{M g D}+\frac{f_{p}(t) l}{M g D} \cos \gamma\right) \\
& -\frac{\omega_{p}^{2} D m \cos \gamma}{M+m \sin ^{2} \gamma}\left(-\frac{c_{p}}{\omega_{p}^{2} m D l} \dot{\gamma}-\frac{l}{D} \sin \gamma+\frac{l}{D} \frac{f_{p}(t)}{m g}\right)+\mathcal{O}(\epsilon) .
\end{aligned}
$$

We have implemented this model in Mathematica to show how a general trajectory $x(t)$ of the full system is attracted to reduced model-trajectories the slow manifold $\mathcal{M}_{\epsilon}$. A graphical illustration of this behavior is shown in Fig. 8 .

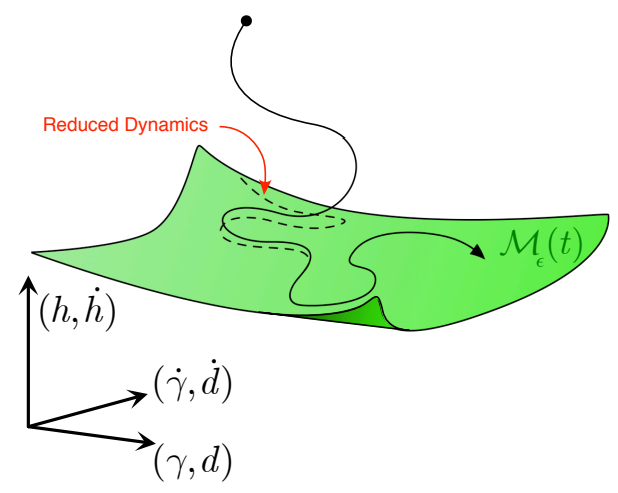

Figure 8: Illustration of the attracting slow manifold $\mathcal{M}_{\epsilon}$ for the mechanical system (56), graphed over the two slow degrees of freedom $x_{\gamma}$ and $x_{d}$ and their corresponding velocities. A general trajectory $q(t)$ is attracted to the slow manifold, synchronizing exponentially fast with a trajectory of reduced dynamics (dashed line).

For a numerical illustration of the accuracy of the reduced model, we choose the following values for the system parameters: 


$$
\begin{gathered}
l=D=6 \mathrm{~m}, \quad L=1 \mathrm{~m}, \quad M=m=1 \mathrm{~kg}, \\
K_{h}=600 \mathrm{~N} / \mathrm{m}, \quad \Gamma_{h}=0.5 \mathrm{~N} / \mathrm{m}^{3}, \quad K_{d}=2 \mathrm{~N} / \mathrm{m}, \\
C_{d}=0.33 \cdot \omega_{p} \cdot M \mathrm{~kg} / \mathrm{s}, \quad C_{h}=3 \cdot \omega_{p} \cdot M \mathrm{~kg} / \mathrm{s}, \\
c_{p}=0.33 \cdot \omega_{p} \cdot m \cdot L^{2}\left(\mathrm{~kg} \cdot \mathrm{m}^{2}\right) / \mathrm{s}, \quad g=9.81 \mathrm{~m} / \mathrm{s}^{2}, \\
f_{p}(t)=0.5 \cdot \sin (t) \mathrm{N}, \quad f_{h}(t)=f_{d}(t)=0.5 \cdot \sin (3 t) \mathrm{N}, \\
\epsilon=1 \cdot 10^{-8} .
\end{gathered}
$$

We give the full system the initial condition

$$
q_{0}=\left(\gamma_{0}, d_{0}, \dot{\gamma}_{0}, \dot{d}_{0}, h_{0}, \dot{h}_{0}\right)=(1.000,1.200,0.000,0.000,0.08182,0.005301),
$$

which lies off the slow manifold $\mathcal{M}_{\epsilon}$, then integrate the trajectory starting from this initial condition in forward time. We track the Euclidean distance between the fast variables $(h(t), \dot{h}(t))$ and the explicitly computable slow manifold $\mathcal{M}_{\epsilon}$ for the given slow variables $(\gamma(t), d(t), \dot{\gamma}(t), \dot{d}(t))$. When the fast variables are $\mathcal{O}\left(10^{-5}\right)$ close to $\mathcal{M}_{\epsilon}$ after the time value $t \geq t_{\epsilon}=15.6 \mathrm{~s}$, we take the point $x_{\epsilon}\left(t_{\epsilon}\right)$ belonging to the full trajectory and use the slow coordinates $\left(\gamma\left(t_{\epsilon}\right), d\left(t_{\epsilon}\right), \dot{\gamma}\left(t_{\epsilon}\right), \dot{d}\left(t_{\epsilon}\right)\right)$ of this point as an initial position for the reduced model (60) and (61). Consecutively, we simulate the reduced model in backward and forward time and compare the results with the results obtained from the full model (see Figs. 9, 10, and 11).

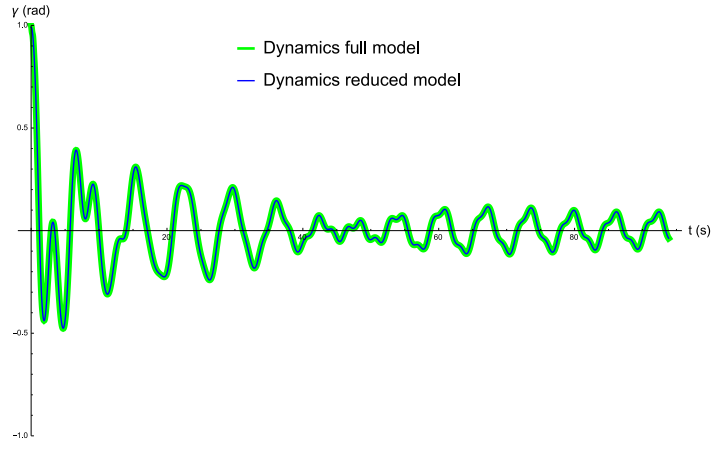

(a)

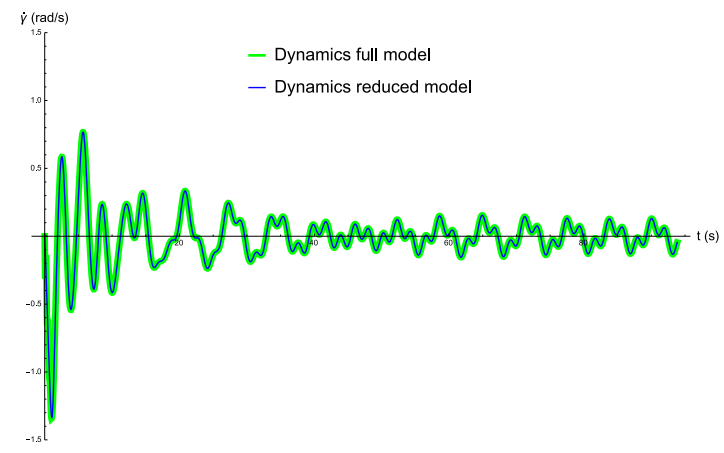

(b)

Figure 9: Exponentially fast synchronization of the soft $(\gamma, \dot{\gamma})$ coordinates of the full trajectory and of a reduced model trajectory. 


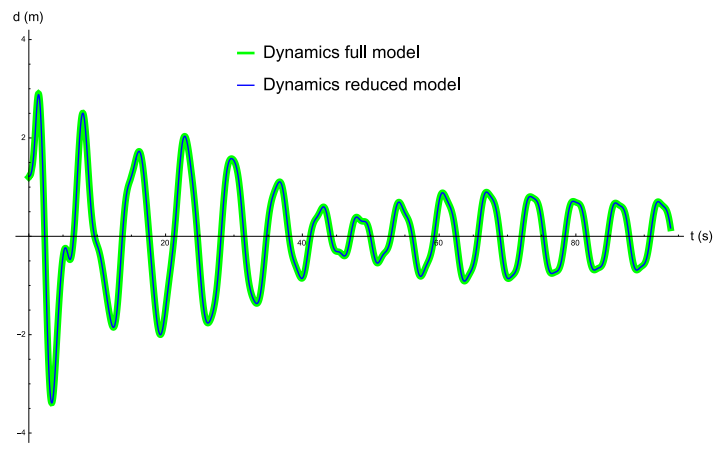

(a)

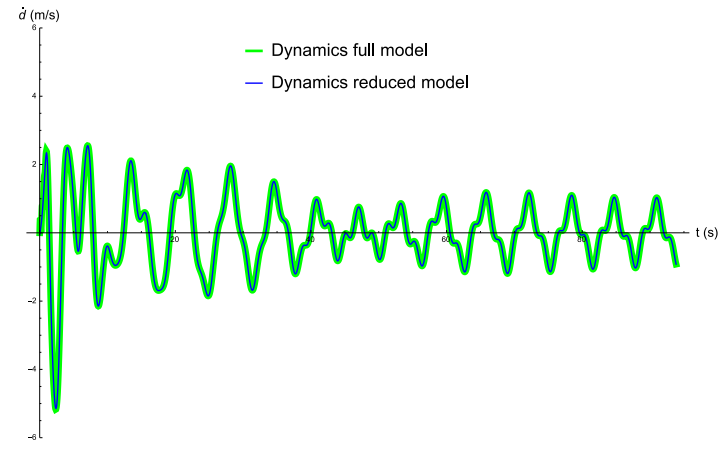

(b)

Figure 10: Exponentially fast synchronization of the soft $(d, \dot{d})$ coordinates of the full trajectory and of a reduced model trajectory.

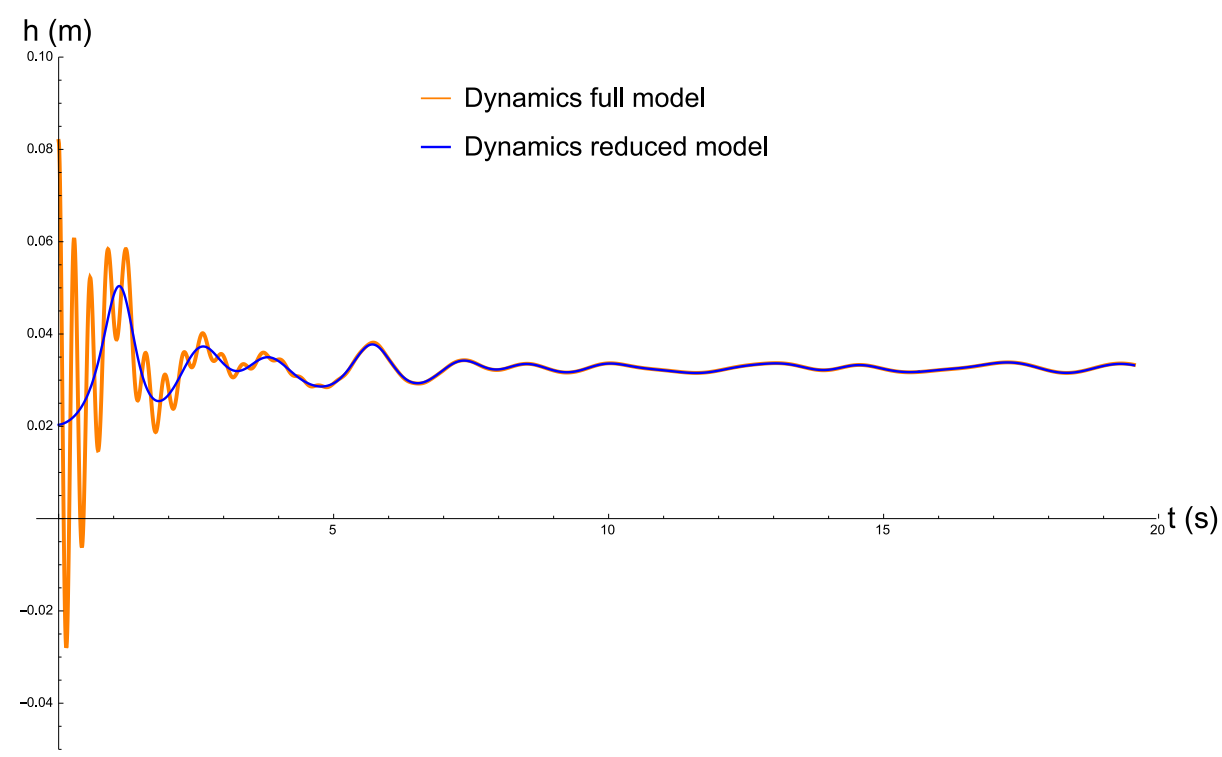

Figure 11: Exponentially fast convergence of the fast coordinate $h$ along the full trajectory to the same coordinate along a trajectory of the reduced system.

\subsection{Two stiff degrees of freedom}

We reconsider here the same mechanical system as in section 6.1. but assume now that both the $d$ and $h$ variables represent stiff degrees of freedom, while $\gamma$ still describes a soft degree of freedom. In this setting, the anticipated slow variable $x$ and fast variable $y=\left(y_{d}, y_{h}\right)$ are defined as

$$
x_{\gamma}=\gamma, \quad y_{d}=d, \quad y_{h}=h,
$$

We express the stiffness of the $y$ degree of freedom by letting

$$
\begin{gathered}
\Delta=\frac{l}{L}=\frac{\delta}{\epsilon}, \quad q_{h}=\frac{\omega_{h}^{2}}{\omega_{p}^{2}}=\frac{\Omega_{h}^{2}}{\epsilon^{2}}, \quad q_{d}=\frac{\omega_{d}^{2}}{\omega_{p}^{2}}=\frac{\Omega_{d}^{2}}{\epsilon^{2}}, \quad a_{h}=\frac{\alpha_{h}}{\epsilon^{4}}, \\
\pi_{h}=\frac{C_{h}}{\omega_{p} M}=\frac{\mu_{h}}{\epsilon}, \quad \pi_{d}=\frac{C_{d}}{\omega_{p} M}=\frac{\mu_{d}}{\epsilon}, \quad \pi_{p}=\frac{c_{p}}{\omega_{p} m L^{2}}=\frac{c_{p}}{\omega_{p} m\left(\frac{\epsilon}{\delta} l\right)^{2}}=\frac{c_{p} \delta^{2}}{\omega_{p} m \epsilon^{2} l^{2}}=\frac{\mu_{p}}{\epsilon^{2}} .
\end{gathered}
$$


As we show in Appendix 12 , assumptions (A1)-(A3) are satisfied in the parameter regime represented by the above scaling for $0<\epsilon \ll 1$. In the scaled variables, we have

$$
M_{1}=\frac{\delta^{2}}{\epsilon^{2}(1+\beta)},
$$

thus the mass matrix $M_{1}$ associated with the slow degree of freedom is not differentiable at $\epsilon=0$. Therefore, only the more general form 18 of the reduced model is applicable, giving

$$
\begin{aligned}
\ddot{x} & =P_{1}\left(x, \dot{x}, G_{0}(x, \dot{x}, t), 0, t ; 0\right)+\mathcal{O}(\epsilon) \\
& =-\frac{\mu_{p}}{\delta^{2}} \dot{x}-\sin x+G_{p}(t)+\mathcal{O}(\epsilon) .
\end{aligned}
$$

Scaling back to the original time, we conclude that at leading order, the exact reduced-order model on the two-dimensional, attracting slow manifold $\mathcal{M}_{\epsilon}$ is given by

$$
\ddot{x}+\frac{\mu_{p}}{\delta^{2}} \dot{x}+\sin x=G_{p}(t)+\mathcal{O}(\epsilon)
$$

or, equivalently,

$$
m l^{2} \ddot{x}+c_{p} \dot{x}+m g l \sin x=f_{p}(t) l+\mathcal{O}(\epsilon) .
$$

As for the example treated in section 6.1. we illustrate numerically that trajectories of the full system synchronize exponentially fast with those of the reduced-order model. For the parameter values

$$
\begin{gathered}
l=6 \mathrm{~m}, \quad L=3 \mathrm{~m}, \quad M=0.25 \mathrm{~kg}, \quad m=0.5 \mathrm{~kg}, \\
K_{h}=2000 \mathrm{~N} / \mathrm{m}, \quad \Gamma_{h}=0.5 \mathrm{~N} / \mathrm{m}^{3}, \quad K_{d}=280 \mathrm{~N} / \mathrm{m}, \\
C_{d}=3 \cdot \omega_{p} \cdot M \mathrm{~kg} / \mathrm{s}, \quad C_{h}=3 \cdot \omega_{p} \cdot M \mathrm{~kg} / \mathrm{s}, \\
c_{p}=\omega_{p} \cdot m \cdot L^{2}\left(\mathrm{~kg} \cdot \mathrm{m}^{2}\right) / \mathrm{s}, \quad g=9.81 \mathrm{~m} / \mathrm{s}^{2}, \\
f_{p}(t)=0.6 \cdot \sin \left(\omega_{p} t\right), \quad f_{h}(t)=f_{d}(t)=0, \\
\epsilon=1 \cdot 10^{-8},
\end{gathered}
$$

and the initial condition,

$$
x_{0}=\left(\gamma_{0}, \dot{\gamma}_{0}, h_{0}, d_{0}, \dot{h}_{0}, \dot{d}_{0}\right)=(1.000,0.000,0.002842,0.02296,0.0005551,-0.002546),
$$

we illustrate the convergence of the trajectory to a trajectory of the reduced model on the slow manifold in Fig. 12p. The target model trajectory was identified as earlier in the soft-soft-stiff version of the same example. 


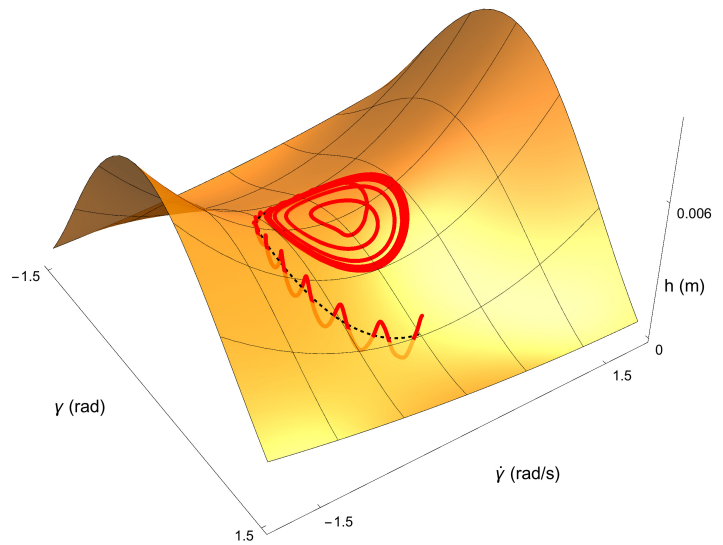

(a)

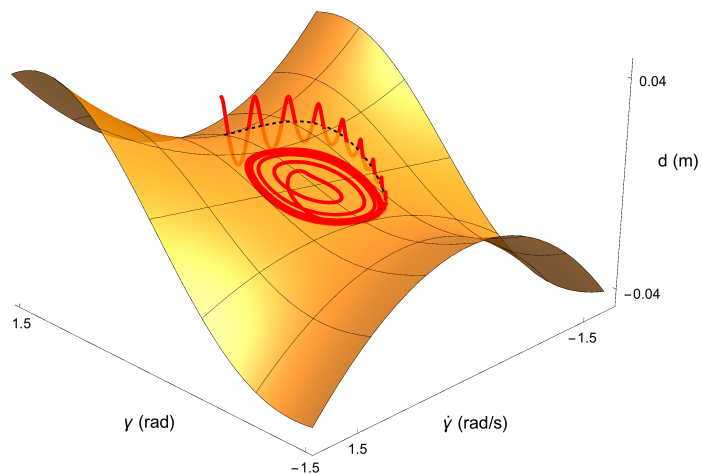

(b)

Figure 12: Instantaneous projection of the slow manifold $\mathcal{M}_{\epsilon}$ for the periodically forced, stiffstiff-soft mechanical system at $t=20 \mathrm{~s}$. 12a A trajectory of the full system is launched at the initial condition $x_{0}=(1.000,0.000,0.002842,0.02296,0.0005551,-0.002546)$ and integrated in forward time. Displayed in red is the $h$ component of the corresponding full system trajectory that converges to the slow manifold and synchronizes with the reduced order model (dashed), shown up to time $t_{\epsilon}=15.6 \mathrm{~s}$. (12b) Convergence of the horizontal coordinate $d$ (red) to the slow manifold, synchronizing with the dynamics of the reduced order model (dashed).

\section{Conclusions}

We have developed a methodology for exact model reduction in multi-degree-of-freedom mechanical systems with soft and stiff degrees of freedom. This Slow-Fast Decomposition (SFD) approach allows for a systematic identification of parameter regimes in which an attracting slow manifold exists. On this invariant manifolds, the stiff variables are enslaved to the remaining soft variables.

We have derived explicit expressions for the slow manifold and for the first two orders of the reduced flow on this manifold. The latter formulas provide a mathematically exact reduced ordermodel with which trajectories of the full system synchronize at an a priori predictable exponential rate. We have also identified a domain boundary over which the slow manifold generically loses its stability and hence the dynamics on it no longer serves as a reduced-order model for the mechanical system.

Slow-fast reduction has previously been carried out with varying levels of mathematical rigor in several specific mechanical model problems (see the Introduction for a review). Our contributions here are: (i) explicit conditions under which an attracting slow manifold in guaranteed to exist in a general, multi-degree-of-freedom mechanical system; (ii) readily applicable general formulas for reduced-order models on such manifolds. All these results follow from the application of classic results from geometric singular perturbation theory (see, e.g., Fenichel [8], Jones [20]).

We have found that the SFD conditions yield reduced-order models that satisfy the basic requirements (R1)-(R2) we have formulated for a mathematically exact model reduction procedure. As we has shown explicitly in Section 5 , the formal methods of static condensation and modal derivatives in structural dynamics can only be justified if the conditions of SFD are satisfied. When these conditions do not hold, the reduced-order models produced by these methods are inaccurate or even qualitatively incorrect.

Importantly, the SFD approach does not require the explicit identification of eigenfrequencies and normal modes for a linearized system, which is a numerically costly undertaking for high-degreeof-freedom systems. Instead, the SFD can be carried out based on a general identification of stiff 
and soft vibratory modes, without an explicit decoupling of these modes. This flexibility for the method enables its application in structural vibrations problems such as those including forced and damped beams (cf. Jain et al. [19] for a detailed example involving the von Kármán beam model).

An extension of the SFD methodology to stiff-soft continuum vibrations described by partial differential equations should also be possible through an appropriate extension of the necessary geometric singular perturbation results to infinite dimensions (see, e.g., Menon and Haller [26]).

\section{Acknowledgments}

We would like to thank Paolo Tiso, Daniel Rixen and Shobhit Jain for useful conversations and for their insights on the subject of this paper.

\section{Appendix : Proof of the main result}

\subsection{First-order autonomous form}

By the nondegeneracy of $M$, the matrices $M_{11}$ and $M_{22}$ are necessarily invertible, which enables us to split (1) in the form

$$
\begin{aligned}
& {\left[M_{11}-M_{12} M_{22}^{-1} M_{21}\right] \ddot{x}=-F_{1}-M_{12} M_{22}^{-1} F_{2},} \\
& {\left[M_{22}-M_{21} M_{11}^{-1} M_{12}\right] \ddot{y}=-F_{2}-M_{21} M_{11}^{-1} F_{1} .}
\end{aligned}
$$

The nondegeneracy of $M$ also implies that the two matrices on the left-hand side of this system must be invertible, leading to the explicit second-order dynamical system

$$
\begin{aligned}
\ddot{x} & =M_{1}^{-1}\left(x, \frac{y}{\epsilon}, t ; \epsilon\right) Q_{1}\left(x, \dot{x}, \frac{y}{\epsilon}, \dot{y}, t ; \epsilon\right), \\
\ddot{y} & =M_{2}^{-1}\left(x, \frac{y}{\epsilon}, t ; \epsilon\right) Q_{2}\left(x, \dot{x}, \frac{y}{\epsilon}, \dot{y}, t ; \epsilon\right),
\end{aligned}
$$

with $M_{i}$ and $Q_{i}$ defined in (5).

In order to convert this system into a first-order autonomous system, we first introduce a phase variable $\varphi \in \mathcal{C}$ such that

$$
\mathcal{C}= \begin{cases}S^{1}, & M_{i}, Q_{i} \text { are periodic in } t \\ \mathbb{T}^{k}, & M_{i}, Q_{i} \text { are quasi-periodic with } k \text { independent frequencies in } t, \\ {[a, b],} & M_{i}, Q_{i} \text { are aperiodic in } t .\end{cases}
$$

We then let

$$
v=\dot{x}, \quad w=\dot{y},
$$

and rewrite equation (64) as a first-order autonomous system on the extended phase space $\mathcal{P}=$ $\mathbb{R}^{s} \times \mathbb{R}^{s} \times \mathbb{R}^{f} \times \mathbb{R}^{f} \times \mathcal{C}$ in the form

$$
\begin{aligned}
\dot{x} & =v, \\
\dot{v} & =M_{1}^{-1}\left(x, \frac{y}{\epsilon}, \varphi ; \epsilon\right) Q_{1}\left(x, v, \frac{y}{\epsilon}, w, \varphi ; \epsilon\right), \\
\dot{y} & =w, \\
\dot{w} & =M_{2}^{-1}\left(x, \frac{y}{\epsilon}, \varphi ; \epsilon\right) Q_{2}\left(x, v, \frac{y}{\epsilon}, w, \varphi ; \epsilon\right), \\
\dot{\varphi} & =\omega,
\end{aligned}
$$

where

$$
\omega= \begin{cases}\omega_{1}, & \mathcal{C}=S^{1} \\ \left(\omega_{1}, \ldots, \omega_{k}\right), & \mathcal{C}=\mathbb{T}^{k} \\ 1, & \mathcal{C}=[a, b]\end{cases}
$$




\subsection{Time-scale separation}

Up to this point, the splitting $q=(x, y)$ has been arbitrary. We now seek conditions under which the $x$-degrees of freedom serve as coordinates for a reduced-order model. For such a reduced-order model to capture effectively the long-term system dynamics, we require the $y$ variables to become enslaved to the $x$ variables and to the phase variable $\varphi$ over a time scale that is an order of magnitude faster than the characteristic time scale of the reduced-order model (cf. the requirement (R2) in the Introduction). To this end, we introduce a characteristic fast time scale $\tau$ by letting $t=\epsilon \tau$, with small, non-dimensional parameter $0<\epsilon \ll 1$. Denoting differentiation with respect to $\tau$ by prime, we obtain the rescaled equations

$$
\begin{aligned}
x^{\prime} & =\epsilon v, \\
v^{\prime} & =\epsilon M_{1}^{-1}\left(x, \frac{y}{\epsilon}, \varphi ; \epsilon\right) Q_{1}\left(x, v, \frac{y}{\epsilon}, w, \varphi ; \epsilon\right), \\
\varphi^{\prime} & =\epsilon \omega, \\
y^{\prime} & =\epsilon w, \\
W^{\prime} & =\epsilon M_{2}^{-1}\left(x, \frac{y}{\epsilon}, \varphi ; \epsilon\right) Q_{2}\left(x, v, \frac{y}{\epsilon}, w, \varphi ; \epsilon\right) .
\end{aligned}
$$

In this new scale, the evolution in the $(y, w)$ variables should be taking place at an $\mathcal{O}(1)$ speed with respect to $\epsilon$, whereas the $(x, v)$ variables should experience an $\mathcal{O}(\epsilon)$ rate of change. By the structure of system (65), this time-scale separation will only arise if we localize $y$ by letting $y=\epsilon \eta$. With this scaling, we obtain the equations

$$
\begin{aligned}
x^{\prime} & =\epsilon v, \\
v^{\prime} & =\epsilon M_{1}^{-1}(x, \eta, \varphi ; \epsilon) Q_{1}(x, v, \eta, w, \varphi ; \epsilon), \\
\varphi^{\prime} & =\epsilon \omega, \\
\eta^{\prime} & =w, \\
w^{\prime} & =\epsilon M_{2}^{-1}(x, \eta, \varphi ; \epsilon) Q_{2}(x, v, \eta, w, \varphi ; \epsilon) .
\end{aligned}
$$

To ensure that $w$ also varies at $\mathcal{O}(1)$ speeds for small enough $\epsilon$, the function $\epsilon M_{2}^{-1}(x, \eta, \varphi ; \epsilon) Q_{2}(x, v, \eta, w, \varphi ; \epsilon)$ must have a smooth, $\mathcal{O}(1)$ limit as $\epsilon \rightarrow 0$. We, therefore, must require the function

$$
P_{2}(x, v, \eta, w, \varphi ; \epsilon)=\epsilon M_{2}^{-1}(x, \eta, \varphi ; \epsilon) Q_{2}(x, v, \eta, w, \varphi ; \epsilon)
$$

to have a smooth limit at $\epsilon=0$, defined by a smooth function

$$
P_{2}(x, v, \eta, w, \varphi ; 0):=\lim _{\epsilon \rightarrow 0} P_{2}(x, v, \eta, w, \varphi ; \epsilon)
$$

on an open and bounded subset of the extended phase space $\mathcal{P}$. In order to be able to carry out a perturbation argument from this limit, we also require that

$$
P_{1}(x, v, \eta, w, \varphi ; \epsilon)=M_{1}^{-1}(x, \eta, \varphi ; \epsilon) Q_{1}(x, v, \eta, w, \varphi ; \epsilon)
$$

has a similar smooth limit at $\epsilon=0$, defined as

$$
P_{1}(x, v, \eta, w, \varphi ; 0):=\lim _{\epsilon \rightarrow 0} P_{1}(x, v, \eta, w, \varphi ; \epsilon) .
$$

With these quantities and assumptions, 66 becomes

$$
\begin{aligned}
x^{\prime} & =\epsilon v, \\
v^{\prime} & =\epsilon P_{1}(x, v, \eta, w, \varphi ; \epsilon), \\
\varphi^{\prime} & =\epsilon \omega, \\
\eta^{\prime} & =w, \\
w^{\prime} & =P_{2}(x, v, \eta, w, \varphi ; \epsilon) .
\end{aligned}
$$




\subsection{Existence of a critical manifold}

We want to ensure the existence of a reduced-order model in which the $(\eta(t), w(t))$ dynamics can be uniquely expressed, at least for large enough times, as a function of the $(x(t), v(t))$ dynamics and the time $t$. In geometric terms, this amounts to the existence of an invariant manifold $\mathcal{M}_{\epsilon}$ that is a graph over the $(x, v, t)$ variables and attracts all nearby solutions of the full system.

We require our reduced model to be smooth in $\epsilon$, which is equivalent to requiring a smooth limit $\mathcal{M}_{0}=\lim _{\epsilon \rightarrow 0} \mathcal{M}_{\epsilon}$ for the invariant manifold in the $\epsilon=0$ limit of system (68). This limiting system can be written as

$$
\begin{aligned}
x^{\prime} & =0, \\
v^{\prime} & =0, \\
\varphi^{\prime} & =0, \\
\eta^{\prime} & =w, \\
w^{\prime} & =P_{2}(x, v, \eta, w, \varphi ; 0) .
\end{aligned}
$$

In this limit, therefore, $(x, v, \varphi) \equiv\left(x_{0}, v_{0}, \varphi_{0}\right)$ plays the role of a constant parameter vector. Any trajectory of the fast dynamics

$$
\begin{aligned}
\eta^{\prime} & =w, \\
w^{\prime} & =P_{2}\left(x_{0}, v_{0}, \eta, w, \varphi_{0} ; 0\right),
\end{aligned}
$$

therefore, gives rise to a $(2 s+1)$-dimensional invariant manifold for the full system. Along nontrivial trajectories of $(70)$, however, the $(\eta, v)$ variables change and hence are not uniquely enslaved to $\left(x_{0}, v_{0}, \varphi_{0}\right)$, as required for the smooth limit of a reduced-order model. Consequently, only invariant manifolds arising from fixed points of $(70)$ can be considered as limits of reduced-order models.

Such fixed points of 70 form a set

$$
\mathcal{M}_{0}=\left\{(x, v, \eta, w, \varphi) \in \mathcal{P}: w=0, \quad P_{2}(x, v, \eta, w, \varphi ; 0)=0\right\} .
$$

To be a limit of a slow manifold carrying a reduced-order model, $\mathcal{M}_{0}$ must be a smooth graph over an open domain $\mathcal{D}_{0} \subset \mathbb{R}^{m} \times \mathbb{R}^{m} \times \mathcal{C}$ of the space $(x, v, t)$ variables. By the implicit function theorem, this is equivalent to the requirement that

$$
\operatorname{det}\left[\partial_{\eta} P_{2}(x, v, \eta, 0, \varphi ; 0)\right] \neq 0,
$$

should hold at all points $(x, v, \eta, w, \varphi) \in \mathcal{M}_{0}$. This condition ensures that if $\mathcal{M}_{0}$ is nonempty, then it is a $2 s+1$ dimensional differentiable manifold that can locally be expressed as a smooth graph

$$
\left(\begin{array}{c}
\eta \\
w
\end{array}\right)=\left(\begin{array}{c}
G_{0}(x, v, \varphi) \\
0
\end{array}\right), \quad(x, v, \varphi) \in \mathcal{D}_{0}
$$

with the function $G_{0}: \mathcal{D}_{0} \rightarrow \mathbb{R}^{f}$ satisfying the identity

$$
P_{2}\left(x, v, G_{0}(x, v, \varphi), 0, \varphi ; 0\right)=0 .
$$

We refer to the part of $\mathcal{M}_{0}$ satisfying (71) as the critical manifold associated with the limiting system (69). In our discussion of assumption (A2), we use the term critical manifold for the $t=$ const. times slice $\mathcal{M}_{0}(t)$ of $\mathcal{M}_{0}$.

\subsection{Stability of $\mathcal{M}_{0}$}

The critical manifold must be normally attracting to persist as an attracting invariant slow manifold in the full system (68). The stability type of $\mathcal{M}_{0}$ can be identified by analyzing the linearization of the fast flow 70 at the fixed points forming $\mathcal{M}_{0}$. 
The stability of the manifold $\mathcal{M}_{0}$ at the fixed point family $\left(\eta_{0}, w_{0}\right)=\left(G\left(x_{0}, v_{0}, \varphi_{0}\right), 0\right)$ of the decoupled equations is governed by the eigenvalues of the Jacobian

$$
J=\left[\begin{array}{cc}
0 & I \\
\partial_{\eta} P_{2} & \partial_{w} P_{2}
\end{array}\right]_{(x, v, \eta, w, t ; \epsilon)=\left(x_{0}, v_{0}, G_{0}\left(y_{0}, w_{0}, \varphi_{0}\right), 0, \varphi_{0} ; 0\right)} .
$$

The matrix $J$ has eigenvalues with strictly negative real parts precisely when the fixed point of the linear vibratory system

$$
u^{\prime \prime}-\partial_{w} P_{2}\left(x_{0}, v_{0}, G_{0}\left(x_{0}, v_{0}, t_{0}\right), 0, \varphi_{0} ; 0\right) u^{\prime}-\partial_{\eta} P_{2}\left(x_{0}, v_{0}, G_{0}\left(x_{0}, v_{0}, \varphi_{0}\right), 0, \varphi_{0} ; 0\right) u=0
$$

is asymptotically stable for the parameter values $\left(x_{0}, v_{0}, \varphi_{0}\right) \in \mathcal{D}_{0}$, which is guaranteed by assumption (A3). In that case, a compact subset of the critical manifold $\mathcal{M}_{0}$ is a compact normally hyperbolic invariant manifold with boundary when $\left(x_{0}, v_{0}, \varphi_{0}\right)$ is restricted to a domain with a smooth boundary. (In case of $\mathcal{C}=[a, b]$, one has to select $a$ and $b$ as smooth functions of $\left(y_{0}, t_{0}\right)$ to eliminate non-smooth corners in $\partial \mathcal{M}_{0}$. This can always be done without loss of generality.)

\subsection{Existence of a slow manifold}

Under the above conditions, the results of Fenichel [8] guarantee for the full system (68) the existence of an attracting slow manifold $\mathcal{M}_{\epsilon}$ that is $\mathcal{O}(\epsilon) C^{r}$-close to $\mathcal{M}_{0}$, and hence continues to be a graph of the form

$$
\left(\begin{array}{c}
\eta \\
w
\end{array}\right)=\left(\begin{array}{c}
G_{\epsilon}(x, v, \varphi) \\
\epsilon H_{\epsilon}(x, v, \varphi)
\end{array}\right)=\left(\begin{array}{c}
G_{0}(x, v, \varphi)+\epsilon G_{1}(x, w, \varphi)+\mathcal{O}\left(\epsilon^{2}\right) \\
\epsilon H_{0}(x, v, \varphi)+\epsilon^{2} H_{1}(x, v, \varphi)+\mathcal{O}\left(\epsilon^{3}\right)
\end{array}\right), \quad(x, v, \varphi) \in \mathcal{D}_{0}
$$

with appropriate smooth functions $G_{\epsilon}$ and $H_{\epsilon}$. The relation $\eta^{\prime}=w$ in 68 imposes the relationships

$$
\begin{aligned}
\frac{d}{d \tau}\left[G_{0}(x, v, \varphi)+\epsilon G_{1}(x, v, \varphi)+\mathcal{O}\left(\epsilon^{2}\right)\right] & =\epsilon H_{0}(x, v, \varphi)+\epsilon^{2} H_{1}(x, v, \varphi)+\mathcal{O}\left(\epsilon^{3}\right), \\
\frac{d}{d \tau}\left[\epsilon H_{0}(x, v, \varphi)+\epsilon^{2} H_{1}(x, v, \varphi)+\mathcal{O}\left(\epsilon^{3}\right)\right] & =P_{2}\left(x, v, G_{\epsilon}(x, v, \varphi), \epsilon H_{\epsilon}(x, v, \varphi), \varphi ; \epsilon\right) .
\end{aligned}
$$

Carrying out the differentiation in these two equations gives

$$
\begin{gathered}
\left(\epsilon \partial_{x} G_{0}+\epsilon^{2} \partial_{x} G_{1}\right) v+\left(\epsilon \partial_{v} G_{0}+\epsilon^{2} \partial_{v} G_{1}\right) P_{1}\left(x, v, G_{\epsilon}, \epsilon H_{\epsilon}, \varphi ; \epsilon\right)+\left(\epsilon \omega \partial_{\varphi} G_{0}+\epsilon^{2} \omega \partial_{\varphi} G_{1}\right)+\mathcal{O}\left(\epsilon^{3}\right) \\
=\epsilon H_{0}+\epsilon^{2} H_{1}+\mathcal{O}\left(\epsilon^{3}\right) \\
\left(\epsilon^{2} \partial_{x} H_{0}+\epsilon^{3} \partial_{x} H_{1}\right) v+\left(\epsilon^{2} \partial_{v} H_{0}+\epsilon^{3} \partial_{v} H_{1}\right) P_{1}\left(x, v, G_{\epsilon}, \epsilon H_{\epsilon}, \varphi ; \epsilon\right)+\left(\epsilon^{2} \omega \partial_{\varphi} H_{0}+\epsilon^{3} \omega \partial_{\varphi} H_{1}\right)+\mathcal{O}\left(\epsilon^{4}\right) \\
=P_{2}\left(x, v, G_{\epsilon}(x, v, \varphi), \epsilon H_{\epsilon}(x, v, \varphi), \varphi ; \epsilon\right)
\end{gathered}
$$

We Taylor-expand these two equations, then equate the $\mathcal{O}(\epsilon)$ and $\mathcal{O}\left(\epsilon^{2}\right)$ terms in the first equation, as well as $\mathcal{O}(\epsilon)$ terms in the second equation, to obtain

$$
\begin{aligned}
H_{0}(x, v, \varphi)= & \partial_{x} G_{0}(x, v, \varphi) v+\partial_{v} G_{0}(x, v, \varphi) P_{1}\left(x, v, G_{0}(x, v, \varphi), 0, \varphi ; 0\right)+\omega \partial_{\varphi} G_{0}(x, v, \varphi), \\
H_{1}(x, w, \varphi)= & \partial_{x} G_{1}(x, v, \varphi) v+\partial_{v} G_{1}(x, v, \varphi) P_{1}\left(x, v, G_{0}(x, v, \varphi), 0, \varphi ; 0\right)+\omega \partial_{\varphi} G_{1}(x, v, \varphi), \\
G_{1}(x, v, \varphi)= & -\left[D_{\eta} P_{2}\left(x, v, G_{0}(x, v, \varphi), 0, \varphi ; 0\right)\right]^{-1} D_{w} P_{2}\left(x, v, G_{0}(x, v, \varphi), 0, \varphi ; 0\right) H_{0}(x, v, \varphi) \\
& -\left[D_{\eta} P_{2}\left(x, v, G_{0}(x, v, \varphi), 0, \varphi ; 0\right)\right]^{-1} D_{\epsilon} P_{2}\left(x, v, G_{0}(x, v, \varphi), 0, \varphi ; 0\right) .
\end{aligned}
$$

In terms of the original variables, therefore, the slow manifold satisfies

$$
\begin{aligned}
& y=\epsilon G_{0}(x, \dot{x}, t)+\epsilon^{2} G_{1}(x, \dot{x}, t)+\mathcal{O}\left(\epsilon^{3}\right), \\
& \dot{y}=\epsilon H_{0}(x, \dot{x}, t)+\epsilon^{2} H_{1}(x, \dot{x}, t)+\mathcal{O}\left(\epsilon^{3}\right),
\end{aligned}
$$

where the functions $H_{0}, G_{1}$ and $H_{1}$ are those listed in (17). 


\subsection{The reduced flow on the slow manifold}

The slow manifold $\mathcal{M}_{\epsilon}$ attracts all nearby solutions, thus the reduced flow on $\mathcal{M}_{\epsilon}$ will serve as the type of reduced-order model we have been seeking to construct (cf. requirement (R1) in the Introduction). The reduced equations on $\mathcal{M}_{\epsilon}$ can be written by restricting the $(x, v, \phi)$ components of our system (68) to $\mathcal{M}_{\epsilon}$, which yields

$$
\begin{aligned}
x^{\prime}= & \epsilon v, \\
v^{\prime}= & \epsilon P_{1}\left(x, v, G_{0}(x, v, \varphi), 0, \varphi ; 0\right) \\
& \quad+\epsilon^{2}\left[D_{\eta} P_{1}\left(x, v, G_{0}(x, v, \varphi), 0, \varphi ; 0\right) G_{1}(x, v, \varphi)+D_{w} P_{1}\left(x, v, G_{0}(x, v, \varphi), 0, \varphi ; 0\right) H_{0}(x, v, \varphi)\right. \\
\quad & \left.\quad+D_{\epsilon} P_{2}\left(x, v, G_{0}(x, v, \varphi), 0, \varphi ; 0\right)\right]+\mathcal{O}\left(\epsilon^{2}\right), \\
\varphi^{\prime}= & \epsilon \omega .
\end{aligned}
$$

In the original set of coordinates, this reduced flow can be written as in eq. (18).

Using the definition of $P_{1}$, we find that if $M_{1}\left(x, G_{0}(x, \dot{x}, t), t\right)$ has a smooth limit at $\epsilon=0$, then the reduced equation can be multiplied by $M_{1}\left(x, G_{0}(x, \dot{x}, t), t\right)$ to yield the leading-order equivalent form of (18) as given in eq. (19). When necessary, the $\mathcal{O}(\epsilon)$ terms in 19 can also be computed from the formulas we have given above.

\subsection{Convergence to the reduced trajectories}

By the invariant foliation results of Fenichel [8], for small enough $\epsilon$ and for motions close enough to the critical manifold, the $y(t)$ component of all solutions of equation (1) synchronize exponentially fast with solutions of the reduced-order model (18).

Specifically, the local stable manifold $W_{\text {loc }}^{s}\left(\mathcal{M}_{\epsilon}\right)$ is foliated by an invariant family of class $C^{r}$ stable fibers $f^{s}(p)$. This $(2 s+\operatorname{dim} \mathcal{C})$-parameter fiber-family is parametrized by the base points $p \in \mathcal{M}_{\epsilon}$ of the fibers. Each fiber is a class $C^{r-1}$ manifold whose dimension is $2 f$. The invariance of the fiber family means that for the flow map $F^{\tau}: \mathcal{P} \rightarrow \mathcal{P}$ of system [68), we have

$$
F^{\tau}\left(f^{s}(p)\right) \subset f^{s}\left(F^{\tau}(p)\right)
$$

for all $\tau>0$. Furthermore, the trajectory of the reduced flow through a fiber base point $p$ attracts exponentially all trajectories that cross the fiber $f^{s}(p)$. Specifically, if $p=\left(x_{R}\left(\tau_{0}\right), v_{R}\left(\tau_{0}\right), \varphi_{R}\left(\tau_{0}\right)\right)$ and $\left(x\left(\tau_{0}\right), v\left(\tau_{0}\right), \varphi\left(\tau_{0}\right), \eta\left(\tau_{0}\right), w\left(\tau_{0}\right)\right) \in f^{s}(p)$, then for all $\tau$ values satisfying

$$
(x(\tau), v(\tau), \varphi(\tau), \eta(\tau), w(\tau)) \in W_{l o c}^{s}\left(\mathcal{M}_{\epsilon}\right),
$$

we have the estimate

$$
\left|\left(\begin{array}{c}
x(\tau)-x_{R}(\tau) \\
v(\tau)-v_{R}(\tau) \\
\varphi(\tau)-\varphi_{R}(\tau) \\
\eta(\tau)-\eta_{R}(\tau) \\
w(\tau)-w_{R}(\tau)
\end{array}\right)\right| \leq C\left|\left(\begin{array}{c}
x\left(\tau_{0}\right)-x_{R}\left(\tau_{0}\right) \\
v\left(\tau_{0}\right)-v_{R}\left(\tau_{0}\right) \\
\varphi\left(\tau_{0}\right)-\varphi_{R}\left(\tau_{0}\right) \\
\eta\left(\tau_{0}\right)-\eta_{R}\left(\tau_{0}\right) \\
w\left(\tau_{0}\right)-w_{R}\left(\tau_{0}\right)
\end{array}\right)\right| e^{-\Lambda\left(\tau-\tau_{0}\right)}, \quad \tau>\tau_{0} .
$$

Here $\Lambda>0$ can be selected as any constant satisfying

$$
\max _{j \in[1,2 f],(x, v, \varphi) \in \mathcal{D}_{0}} \operatorname{Re} \lambda_{j}(x, v, \varphi)<-\Lambda<0,
$$

with $\lambda_{j}(x, v, \varphi), j=1, \ldots, 2 f$, denoting the eigenvalues of the Jacobian $J$, or equivalently, of the associated linear system 75 . The constant $C>0$ depends on $\Lambda$ but is independent of the choice of the fiber base point $p$ and the times $\tau$ and $\tau_{0}$.

By the form of system system $(68)$, we have $\left|\varphi(\tau)-\varphi_{R}(\tau)\right|=\left|\varphi\left(\tau_{0}\right)-\varphi_{R}\left(\tau_{0}\right)\right|$. This is only consistent with $(76)$, if $\varphi\left(\tau_{0}\right) \equiv \varphi_{R}\left(\tau_{0}\right)$, which implies that the fibers $f^{s}(p)$ are necessarily flat (i.e, 
constant) in the coordinate $\varphi$. Using this fact in 76 and passing back to the original coordinates gives

$$
\left|\left(\begin{array}{c}
x(t)-x_{R}(t) \\
\dot{x}(t)-\dot{x}_{R}(t) \\
\frac{1}{\epsilon} y(t)-\frac{1}{\epsilon} y_{R}(t) \\
\dot{y}(t)-\dot{y}(t)
\end{array}\right)\right| \leq C\left|\left(\begin{array}{c}
x\left(t_{0}\right)-x_{R}\left(t_{0}\right) \\
\dot{x}\left(t_{0}\right)-\dot{x}_{R}\left(t_{0}\right) \\
\frac{1}{\epsilon} y\left(t_{0}\right)-\frac{1}{\epsilon} y_{R}\left(t_{0}\right) \\
\dot{y}\left(t_{0}\right)-\dot{y}_{R}\left(t_{0}\right)
\end{array}\right)\right| e^{-\frac{\Lambda}{\epsilon}\left(t-t_{0}\right)}, \quad \tau>\tau_{0} .
$$

Along the reduced flow on the slow manifold $\mathcal{M}_{\epsilon}$, the $(y, \dot{y})$ variables are enslaved to the $(x, v, t)$ variables, thus we can further rewrite this last inequality as

$$
\left|\left(\begin{array}{c}
x(t)-x_{R}(t) \\
\dot{x}(t)-\dot{x}_{R}(t) \\
\frac{1}{\epsilon} y(t)-G_{\epsilon}\left(x_{R}(t), \dot{x}_{R}(t), t\right) \\
\dot{y}(t)-\epsilon H_{\epsilon}\left(x_{R}(t), \dot{x}_{R}(t), t\right)
\end{array}\right)\right| \leq C\left|\left(\begin{array}{c}
x\left(t_{0}\right)-x_{R}\left(t_{0}\right) \\
\dot{x}\left(t_{0}\right)-\dot{x}_{R}\left(t_{0}\right) \\
\frac{1}{\epsilon} y\left(t_{0}\right)-G_{\epsilon}\left(x_{R}\left(t_{0}\right), \dot{x}_{R}\left(t_{0}\right), t\right) \\
\dot{y}\left(t_{0}\right)-\epsilon H_{\epsilon}\left(x_{R}\left(t_{0}\right), \dot{x}_{R}\left(t_{0}\right), t\right)
\end{array}\right)\right| e^{-\frac{\Lambda}{\epsilon}\left(t-t_{0}\right),} \quad \tau>\tau_{0} .
$$

Applying the triangle inequality to the left-hand-side and using the definition of $G_{\epsilon}$ and $H_{\epsilon}$ on the right-hand side of this inequality proves formula (21).

\section{Appendix: Proof of Proposition 1}

We start by noting that, as a consequence of assumption $(30)$, the graph

$$
\eta=G_{0}(x, t)
$$

of the critical manifold $\mathcal{M}_{0}$ depends only on the slow positions $x$ and the time $t$. Near the unperturbed equilibrium, $\mathcal{M}_{0}(t)$ can therefore be approximated by its Taylor expansion with respect to $x$. Specifically, we have

$$
\eta=G_{0}(x, t)=G_{0}(0, t)+\partial_{x} G_{0}(0, t) x+\frac{1}{2}\left(\partial_{x x}^{2} G_{0}(0, t) x\right) x+\mathcal{O}\left(|x|^{3}\right) .
$$

Differentiation of the implicit equation $P_{2}\left(x, G_{0}(x, t), 0, t ; 0\right)=0$ with respect to $x$ gives

$$
\partial_{x} P_{2}+\partial_{\eta} P_{2} \partial_{x} G_{0}=0 .
$$

Substitution of (77) into(78) and setting $x=0$ yields

$$
\partial_{x} G_{0}(0, t)=-\left.\left[\partial_{\eta} P_{2}\right]^{-1} \partial_{x} P_{2}\right|_{x=0, \eta=G(0, t), \dot{y}=0, \epsilon=0},
$$

where the inverse of $\partial_{\eta} P_{2}\left(x, G_{0}(x, t), 0, t ; 0\right)$ is guaranteed to exist by assumption (A3). Differentiating (78) once more in $x$ gives

$$
\partial_{x x}^{2} P_{2}+\left(2 \partial_{x \eta}^{2} P_{2}+\partial_{\eta \eta}^{2} P_{2} \partial_{x} G_{0}\right) \partial_{x} G_{0}+\partial_{\eta} P_{2} \partial_{x}^{2} G_{0}=0
$$

enabling us to express the three-tensor $\partial_{x x}^{2} G_{0}(0, t)$ as

$$
\partial_{x x}^{2} G_{0}(0, t)=-\left.\left[\partial_{\eta} P_{2}\right]^{-1}\left[\partial_{x x}^{2} P_{2}+\left(2 \partial_{x \eta}^{2} P_{2}+\partial_{\eta \eta}^{2} P_{2} \partial_{x} G_{0}\right) \partial_{x} G_{0}\right]\right|_{x=0, \eta=G(0, t), \dot{y}=0, \epsilon=0} .
$$

Therefore, with the help of the formulas (30), the critical manifold $\mathcal{M}_{0}$ can be written near the origin as a smooth, codimension- $2 f$ graph of the form

$$
\mathcal{M}_{0}(t)=\left\{(x, \dot{x}, \eta, \dot{y}, t) \in \mathcal{P}: \eta=G_{0}(x, t)=\Gamma(t)+\Phi(t) x+(\Theta(t) x) x+\mathcal{O}\left(|x|^{3}\right), \quad \dot{y}=0\right\},
$$


where

$$
\begin{aligned}
P_{2}(0, \Gamma(t), 0, t ; 0) & =0 \\
\Phi(t) & =-\left.\left[\partial_{\eta} P_{2}\right]^{-1} \partial_{x} P_{2}\right|_{x=0, \eta=\Gamma(t), \dot{y}=0, \epsilon=0} \\
\Theta(t) & =-\left.\frac{1}{2}\left[\partial_{\eta} P_{2}\right]^{-1}\left[\partial_{x x}^{2} P_{2}+\left(2 \partial_{x \eta}^{2} P_{2}+\partial_{\eta \eta}^{2} P_{2} \Phi(t)\right) \Phi(t)\right]\right|_{x=0, \eta=\Gamma(t), \dot{y}=0, \epsilon=0}
\end{aligned}
$$

as claimed in statement (i) of the Proposition. These expressions in (81) can then be used in the reduced-order models (18)- $(19)$ to obtain more specific local approximations to the reduced dynamics, in case a global expression for the critical manifold is not explicitly available. Specifically, 18) can be localized near $x=0$ as

$$
\ddot{x}-P_{1}(x, \dot{x},[\Gamma(t)+\Phi(t) x+(\Theta(t) x) x], 0, t ; 0)+\mathcal{O}\left(\epsilon,|x|^{3}\right)=0 .
$$

Under the further assumptions in statement (ii) of the Proposition, we have the following simplifications in formulas $(81)$ :

$$
\Gamma(t) \equiv 0, \quad \Phi(t) \equiv 0, \quad \Theta(t) \equiv-\frac{1}{2}\left[\partial_{\eta} P_{2}(0,0,0 ; 0)\right]^{-1} \partial_{x x}^{2} P_{2}(0,0,0 ; 0) .
$$

Substituting these quantities into 82 and truncating the expression for $\mathcal{M}_{0}(t)$ at linear and then at quadratic order proves the leading-order forms of the reduced equations in statements (iii) and (iv) of the Proposition, respectively. To obtain the order of the error terms in these equations, note that if $x$ and $y$ are modal coordinates of the linearized system, then we have

$$
\begin{aligned}
P_{1}(x, \dot{x}, \eta, \dot{y}, t ; \epsilon) & =P_{1}(x, \dot{x}, \eta, 0, t ; 0)+\mathcal{O}(\epsilon) \\
& =P_{1}(x, \dot{x}, 0,0, t ; 0)+\mathcal{O}(|x||\eta|)+\mathcal{O}(\epsilon) .
\end{aligned}
$$

Substitution of $\eta=0+\mathcal{O}\left(|x|^{2}\right)$ and $\eta=(\Theta(t) x) x+\mathcal{O}\left(|x|^{3}\right)$, respectively, into the $\mathcal{O}(|x||\eta|)$ term in (84) then proves the order of the higher-order terms, as listed in statements (iii) and (iv) of the Proposition.

\section{Appendix: Details for Example 8}

For the system

$$
\begin{aligned}
\ddot{x}+\left(c_{1}+\mu_{1} x^{2}\right) \dot{x}+k_{1} x+a x y+b x^{3} & =0, \quad x \in \mathbb{R}, \\
\ddot{y}+c_{2} \dot{y}+k_{2} y+c x^{2} & =0, \quad y \in \mathbb{R},
\end{aligned}
$$

we consider reduction by static condensation via the linear change of variables

$$
\left(\begin{array}{l}
x \\
y
\end{array}\right)=U \hat{x}, \quad U=\left(\begin{array}{l}
1 \\
0
\end{array}\right), \quad \hat{x} \in \mathbb{R} .
$$

Dropping the tilde from $\hat{x}$ and substituting $y=0$ from 86 into the first equation of 44 gives the statically condensed model 45 .

Next, applying the idea of modal derivatives, we seek a quadratic invariant manifold of the form (32), with the coefficients computed in the unscaled variables as

$$
\begin{aligned}
\Phi & =0 \\
\Theta & =-\left.\frac{1}{2}\left[\partial_{y}\left(c_{2} \dot{y}+k_{2} y+c x^{2}\right)\right]^{-1}\left[\partial_{x x}^{2}\left(c_{2} \dot{y}+k_{2} y+c x^{2}\right)\right]\right|_{x=0, y=0, \dot{y}=0}=-\frac{c}{k_{2}} .
\end{aligned}
$$


Substitution of $y=\Theta x^{2}$ into into the first equation of (44) gives the modal-derivate-based reducedorder model (46), representing only a slight correction to (45) at cubic order. All this appears reasonable at this point, with the statically condensed system 45 offering a leading-order model that is subsequently refined at cubic order by the modal derivatives approach in 46.

At the same time, there exists a slow spectral submanifold (SSM), the unique smoothest, nonlinear continuation of the $y=0$ modal subspace of the equilibrium. This unique, two-dimensional analytic invariant manifold is tangent to the modal subspace of the $x$-degree of freedom at the origin (cf. Haller and Ponsioen [17]). The slow SSM, therefore, can locally be written as a two-dimensional invariant graph $(y, \dot{y})=\left(g_{1}(x, \dot{x}), g_{2}(x, \dot{x})\right)=\mathcal{O}\left(x^{2}, x \dot{x}, \dot{x}^{2}\right)$ over $(x, \dot{x})$, as originally envisioned by Shaw and Pierre [29]. Differentiating the general form

$$
y=g_{1}(x, \dot{x})=\alpha x^{2}+\beta x \dot{x}+\gamma \dot{x}^{2}+\mathcal{O}(3)
$$

of such an invariant graph twice in time, with $\ddot{x}$ substituted from the first equation of system [44], we obtain

$$
\begin{aligned}
\ddot{y}= & -k_{1}\left(2 \alpha-2 \gamma k_{1}-\beta c_{1}\right) x^{2} \\
& -\left[2 \beta k_{1}+c_{1}\left(2 \alpha-2 \gamma k_{1}-\beta c_{1}\right)+2\left(\beta-2 \gamma c_{1}\right) k_{1}\right] x \dot{x} \\
& +\left[\left(2 \alpha-2 \gamma k_{1}-\beta c_{1}\right)-2 c_{1}\left(\beta-2 \gamma c_{1}\right)\right] \dot{x}^{2} \\
& +\mathcal{O}(3) .
\end{aligned}
$$

A comparison of this differential equation with the second equation of system (44), with $y$ and $\dot{y}$ substituted from (88), leads to the linear system of algebraic equations

$$
\left(\begin{array}{ccc}
k_{2}-2 k_{1} & k_{1}\left(c_{1}-c_{2}\right) & 2 k_{1}^{2} \\
2\left(c_{2}-c_{1}\right) & k_{2}-4 k_{1}+c_{1}^{2}-c_{1} c_{2} & 2 k_{1}\left(3 c_{1}-c_{2}\right) \\
2 & c_{2}-3 c_{1} & k_{2}-2 k_{1}+4 c_{1}^{2}-2 c_{1} c_{2}
\end{array}\right)\left(\begin{array}{l}
\alpha \\
\beta \\
\gamma
\end{array}\right)=-\left(\begin{array}{l}
c \\
0 \\
0
\end{array}\right)
$$

for the unknown coefficients $\alpha, \beta$ and $\gamma$ in the expression (88) of the slow SSM. The solution of this system of equations is given by

$$
\begin{aligned}
\alpha & =-\frac{c}{D}\left(4 c_{1}^{4}-6 c_{1}^{3} c_{2}+2 c_{1}^{2} c_{2}^{2}+5 c_{1}^{2} k_{2}-c_{1} c_{2}\left(2 k_{1}+3 k_{2}\right)+2 c_{2}^{2} k_{1}+8 k_{1}^{2}-6 k_{1} k_{2}+k_{2}^{2}\right), \\
\beta & =-\frac{2 c}{D}\left(4 c_{1} k_{1}+k_{2}\left(c_{1}-c_{2}\right)+2 c_{1} c_{2}^{2}-6 c_{1}^{2} c_{2}+4 c_{1}^{3}\right), \\
\gamma & =-\frac{2 c}{D}\left(2 c_{1}^{2}-3 c_{1} c_{2}+c_{2}^{2}+4 k_{1}-k_{2}\right)
\end{aligned}
$$

with

$$
D=\left(c_{1}^{2}-c_{1} c_{2}+k_{2}\right)\left(4 c_{1}^{2} k_{2}-8 c_{1} c_{2} k_{1}-2 c_{1} c_{2} k_{2}+4 c_{2}^{2} k_{1}+16 k_{1}^{2}-8 k_{1} k_{2}+k_{2}^{2}\right) .
$$

With these coefficients, substitution of 88 into the first equation of system (44) gives the exact reduced system on the slow SSM, up to cubic order, in the form

$$
\ddot{x}+\left[c_{1}+\left(\mu_{1}+a \beta\right) x^{2}\right] \dot{x}+\left(k_{1}+a \gamma \dot{x}^{2}\right) x+(b+a \alpha) x^{3}+\mathcal{O}(4)=0 .
$$

Substitution of the formulas 89 into this last equation gives the final form 47 of the exact reduced model on the SSM.

\section{Appendix: Details for Section 6.1}

For the parameter range described by the scalings $[59)$, we take the $\epsilon \rightarrow 0$ limit in the expressions for $P_{1}$ and $P_{2}$ in (57)-(58). We then obtain 


$$
\begin{aligned}
& P_{1}(x, v, \eta, w, t ; 0)=\left[\begin{array}{c}
p_{1}^{0} \\
p_{2}^{0}
\end{array}\right] \\
& p_{1}^{0}=\frac{1+\beta}{\delta^{2}}\left(-\mu_{p} v_{\gamma}-\delta^{2} \sin x_{\gamma}+\delta^{2} G_{p}(t)\right. \\
& \left.+\frac{\delta \sin x_{\gamma}}{1+\beta}\left[\beta \delta \cos x_{\gamma} v_{\gamma}^{2}-\mu_{h} w-\Omega_{h}^{2} \eta-\alpha_{h} \eta^{3}+(1+\beta) \delta+F_{h}(t) \delta-F_{p}(t) \delta \sin x_{\gamma}\right]\right) \\
& -\frac{\phi}{\delta} \cos x_{\gamma}\left(\beta \frac{\delta}{\phi} \sin x_{\gamma} v_{\gamma}^{2}-\mu_{d} v_{d}-\Omega_{d}^{2}\left(1+x_{d}\right) Q^{0}\left(x_{d}\right)+F_{d}(t) \frac{\delta}{\phi}+F_{p}(t) \frac{\delta}{\phi} \cos x_{\gamma}\right), \\
& p_{2}^{0}=-\frac{\beta}{\delta \phi} \cos x_{\gamma}\left(-\mu_{p} v_{\gamma}-\delta^{2} \sin x_{\gamma}+\delta^{2} G_{p}(t)\right. \\
& \left.+\frac{\delta \sin x_{\gamma}}{1+\beta}\left[\beta \delta \cos x_{\gamma} v_{\gamma}^{2}-\mu_{h} w-\Omega_{h}^{2} \eta-\alpha_{h} \eta^{3}+(1+\beta) \delta+F_{h}(t) \delta-F_{p}(t) \delta \sin x_{\gamma}\right]\right) \\
& +\frac{1}{1+\beta}\left(1+\beta \cos ^{2} x_{\gamma}\right)\left(\beta \frac{\delta}{\phi} \sin x_{\gamma} v_{\gamma}^{2}-\mu_{d} v_{d}-\Omega_{d}^{2}\left(1+x_{d}\right) Q^{0}\left(x_{d}\right)+F_{d}(t) \frac{\delta}{\phi}+F_{p}(t) \frac{\delta}{\phi} \cos x_{\gamma}\right), \\
& P_{2}(x, v, \eta, w, t ; 0)=\left(\frac{1+\beta \sin ^{2} x_{\gamma}}{1+\beta}\right)\left(\beta \delta \cos x_{\gamma} v_{\gamma}^{2}-\mu_{h} w-\Omega_{h}^{2} \eta-\alpha_{h} \eta^{3}+(1+\beta) \delta+F_{h}(t) \delta-F_{p}(t) \delta \sin x_{\gamma}\right. \\
& +\frac{(1+\beta) \beta \sin x_{\gamma}}{\delta\left(1+\beta \sin ^{2} x_{\gamma}\right)}\left[-\mu_{p} v_{\gamma}-\delta^{2} \sin x_{\gamma}+\delta^{2} G_{p}(t)\right] \\
& \left.-\frac{\beta \phi \sin x_{\gamma} \cos x_{\gamma}}{1+\beta \sin ^{2} x_{\gamma}}\left[\beta \frac{\delta}{\phi} \sin x_{\gamma} v_{\gamma}^{2}-\mu_{d} v_{d}-\Omega_{d}^{2}\left(1+x_{d}\right) Q^{0}\left(x_{d}\right)+F_{d}(t) \frac{\delta}{\phi}+F_{p}(t) \frac{\delta}{\phi} \cos x_{\gamma}\right]\right),
\end{aligned}
$$

where $Q^{0}\left(x_{d}\right)$ is defined as

$$
Q^{0}\left(x_{d}\right)=\left(1-\frac{1}{1+x_{d}}\right), \quad x_{d}>-1 .
$$

We observe that both $P_{1}$ and $P_{2}$ continue to be smooth in $\epsilon$ at the $\epsilon=0$ limit, thereby satisfying assumption (A1).

For the critical manifold defined through the relationship $\eta=G_{0}(x, v, t)$ in assumption (A2), we have the equation

$$
P_{2}(x, v, \eta, 0, t ; 0)=0 \quad \Longleftrightarrow \quad \Omega_{h}^{2} \eta+\alpha_{h} \eta^{3}=T(x, v, t),
$$

where

$$
\begin{aligned}
T(x, v, t)= & \beta \delta \cos x_{\gamma} v_{\gamma}^{2}+(1+\beta) \delta+F_{h}(t) \delta-F_{p}(t) \delta \sin x_{\gamma} \\
& +\frac{(1+\beta) \beta \sin x_{\gamma}}{\delta\left(1+\beta \sin ^{2} x_{\gamma}\right)}\left[-\mu_{p} v_{\gamma}-\delta^{2} \sin x_{\gamma}+\delta^{2} G_{p}(t)\right] \\
& -\frac{\beta \phi \sin x_{\gamma} \cos x_{\gamma}}{1+\beta \sin ^{2} x_{\gamma}}\left[\beta \frac{\delta}{\phi} \sin x_{\gamma} v_{\gamma}^{2}-\mu_{d} v_{d}-\Omega_{d}^{2}\left(1+x_{d}\right) Q^{0}\left(x_{d}\right)+F_{d}(t) \frac{\delta}{\phi}+F_{p}(t) \frac{\delta}{\phi} \cos x_{\gamma}\right] .
\end{aligned}
$$

Using the cubic formula, the real root of this equation can be expressed explicitly as

$$
\eta=G_{0}(x, v, t)=\sqrt[3]{\frac{T(x, v, t)}{2 \alpha_{h}}+\sqrt{\frac{T^{2}(x, v, t)}{4 \alpha_{h}^{2}}+\frac{\Omega_{h}^{6}}{27 \alpha_{h}^{3}}}}-\sqrt[3]{-\frac{T(x, v, t)}{2 \alpha}+\sqrt{\frac{T^{2}(x, v, t)}{4 \alpha_{h}^{2}}+\frac{\Omega_{h}^{6}}{27 \alpha_{h}^{3}}}},
$$


assuming that $\Omega_{h}^{2}$ and $\alpha_{h}$ are greater than zero.

The oscillatory system (9) determining the stability of the critical manifold takes the specific form

$$
\begin{aligned}
& A(x, v, t)=-\partial_{w} P_{2}\left(x, v, G_{0}(x, v, t), 0, t ; 0\right)=\left(\frac{1+\beta \sin ^{2} x_{\gamma}}{1+\beta}\right) \mu_{h}, \\
& B(x, v, t)=-\partial_{\eta} P_{2}\left(x, v, G_{0}(x, v, t), 0, t ; 0\right)=\left(\frac{1+\beta \sin ^{2} x_{\gamma}}{1+\beta}\right)\left(\Omega_{h}^{2}+3 \alpha_{h} G_{0}^{2}(x, v, t)\right) .
\end{aligned}
$$

The equilibrium solution of the unforced linear oscillatory system 9 is, therefore, always asymptotically stable, given that

$$
\mu_{h}>0, \quad \beta>0, \quad \Omega_{h}^{2}>0, \quad \alpha_{h}>0 .
$$

We conclude that assumptions (A1)-(A3) hold, and hence a global reduced-order model (18) exists over the slow variables in the specific form

$$
\ddot{x}=\left[\begin{array}{l}
\frac{1+\beta}{\delta^{2}\left(1+\beta \sin ^{2} x_{\gamma}\right)} \mathcal{A}-\frac{\phi \cos x_{\gamma}}{\delta\left(1+\beta \sin ^{2} x_{\gamma}\right)} \mathcal{B} \\
\frac{1}{1+\beta \sin ^{2} x_{\gamma}} \mathcal{B}-\frac{\beta \cos x_{\gamma}}{\phi \delta\left(1+\beta \sin ^{2} x_{\gamma}\right)} \mathcal{A}
\end{array}\right]+\mathcal{O}(\epsilon)
$$

where

$$
\begin{aligned}
\mathcal{A}\left(x_{\gamma}, \dot{x}_{\gamma}\right) & =-\mu_{p} \dot{x}_{\gamma}-\delta^{2} \sin x_{\gamma}+\delta^{2} G_{p}(t), \\
\mathcal{B}\left(x_{\gamma}, x_{d}, \dot{x}_{\gamma}\right) & =\beta \frac{\delta}{\phi} \sin x_{\gamma} \dot{x}_{\gamma}^{2}-\mu_{d} \dot{x}_{\gamma}-\Omega_{d}^{2} x_{d}+F_{d}(t) \frac{\delta}{\phi}+F_{p}(t) \frac{\delta}{\phi} \cos x_{\gamma} .
\end{aligned}
$$

Scaling back to the original time and substituting the physical parameters back into the nondimensionalized equations, we obtain that the exact reduced-order model on the slow manifold of the form 60$)-(61)$

\section{Appendix: Details for Section 6.2 .}

Here we verify assumptions (A1)-(A3) in detail for the fast-fast-slow setting treated in Section 6.2 To make the horizontal spring stiff, we choose its length as $D=L$, so that the original equations of motion (54) now become

$$
\begin{aligned}
m l^{2} \ddot{\gamma}-m l \sin \gamma \ddot{h}+m l \cos \gamma \ddot{d}+c_{p} \dot{\gamma}+m g l \sin \gamma & =f_{p}(t) l, \\
(M+m) \ddot{h}-m l \sin \gamma \ddot{\gamma}-m l \cos \gamma \dot{\gamma}^{2}+C_{h} \dot{h}+K_{h} h+K_{d} Q(d, h) h+\Gamma_{h} h^{3} & =(M+m) g+f_{h}(t)-f_{p}(t) \sin \gamma, \\
(M+m) \ddot{d}+m l \cos \gamma \ddot{\gamma}-m l \sin \gamma \dot{\gamma}^{2}+C_{d} \dot{d}+K_{d}(L+d) Q(d, h) & =f_{d}(t)+f_{p}(t) \cos \gamma,
\end{aligned}
$$

with

$$
Q(d, h)=\left(1-\frac{L}{\sqrt{(L+d)^{2}+h^{2}}}\right) .
$$

The linearized oscillation frequencies of the uncoupled springs and pendulum remain the same as in (55). We adopt the same scaling as in section 6.1. except that we now scale the $d$ coordinate with the unstretched length $L$ of the vertical spring. Denoting differentiation with respect to the new time $\tilde{t}$ still by a dot, then dropping all the tildes, we obtain the non-dimensionalized equations of motions 


$$
\begin{aligned}
\Delta^{2} \ddot{\gamma}-\Delta \sin \gamma \ddot{h}+\Delta \cos \gamma \ddot{d}+\pi_{p} \dot{\gamma}+\Delta^{2} \sin \gamma & =\Delta^{2} G_{p}(t), \\
(1+\beta) \ddot{h}-\beta \Delta \sin \gamma \ddot{\gamma}-\beta \Delta \cos \gamma \dot{\gamma}^{2}+\pi_{h} \dot{h}+q_{h} h+q_{d} h Q(d, h)+a_{h} h^{3} & =(1+\beta) \Delta+F_{h}(t) \Delta-F_{p}(t) \Delta \sin \gamma, \\
(1+\beta) \ddot{d}+\beta \Delta \cos \gamma \ddot{\gamma}-\beta \Delta \sin \gamma \dot{\gamma}^{2}+\pi_{d} \dot{d}+q_{d}(1+d) Q(d, h) & =F_{d}(t) \Delta+F_{p}(t) \Delta \cos \gamma .
\end{aligned}
$$

In the notation used for system (1), we now have

$$
\begin{aligned}
M(q, t ; \epsilon)= & \left(\begin{array}{ccc}
\Delta^{2} & -\Delta \sin x & \Delta \cos x \\
-\beta \Delta \sin x & 1+\beta & 0 \\
\beta \Delta \cos x & 0 & 1+\beta
\end{array}\right) \\
F(q, \dot{q}, t ; \epsilon)= & \left(\begin{array}{l}
-\pi_{p} \dot{x}-\Delta^{2} \sin x+\Delta^{2} G_{p}(t) \\
\beta \Delta \cos x \dot{x}^{2}-\pi_{h} \dot{y}_{h}-q_{h} \epsilon \frac{y_{h}}{\epsilon}-q_{d} \epsilon \frac{y_{h}}{\epsilon} Q\left(\frac{y_{d}}{\epsilon}, \frac{y_{h}}{\epsilon}\right)-a_{h} \epsilon^{3}\left(\frac{y_{h}}{\epsilon}\right)^{3} \\
+(1+\beta) \Delta+F_{h}(t) \Delta-F_{p}(t) \Delta \sin x \\
\beta \Delta \sin x \dot{x}^{2}-\pi_{d} \dot{y}_{d}-q_{d}\left(1+\epsilon \frac{y_{d}}{\epsilon}\right) Q\left(\frac{y_{d}}{\epsilon}, \frac{y_{h}}{\epsilon}\right)+F_{d}(t) \Delta+F_{p}(t) \Delta \cos x
\end{array}\right),
\end{aligned}
$$

with the parameter $\epsilon>0$ yet to be determined based on the assumptions of the SFD approach. Note that the mass-matrix above is not symmetric due to the scalings we have employed, but it is nevertheless nonsingular, as we generally assume in this paper.

With the above quantities at hand, we obtain the modified mass matrices $M_{i}$ and the forcing terms $Q_{i}$ defined in (5) in the specific form

$$
\begin{aligned}
M_{1}= & M_{11}-M_{12} M_{22}^{-1} M_{21}=\frac{\Delta^{2}}{1+\beta}, \\
M_{2}= & M_{22}-M_{21} M_{11}^{-1} M_{12}=\left(\begin{array}{cc}
1+\beta \cos ^{2} x & \beta \sin x \cos x \\
\beta \sin x \cos x & 1+\beta \sin ^{2} x
\end{array}\right), \\
Q_{1}= & F_{1}-M_{12} M_{22}^{-1} F_{2}=-\pi_{p} \dot{x}-\Delta^{2} \sin x+\Delta^{2} G_{p}(t)+\frac{\Delta}{1+\beta} \sin x\left[\beta \Delta \cos x \dot{x}^{2}-\pi_{h} \dot{y}_{h}\right. \\
& \left.-q_{h} \epsilon \frac{y_{h}}{\epsilon}-q_{d} \epsilon \frac{y_{h}}{\epsilon} Q\left(\frac{y_{d}}{\epsilon}, \frac{y_{h}}{\epsilon}\right)-a_{h} \epsilon^{3}\left(\frac{y_{h}}{\epsilon}\right)^{3}+(1+\beta) \Delta+F_{h}(t) \Delta-F_{p}(t) \Delta \sin x\right] \\
& -\frac{\Delta}{1+\beta} \cos x\left[\beta \Delta \sin x \dot{x}^{2}-\pi_{d} \dot{y}_{d}-q_{d}\left(1+\epsilon \frac{y_{d}}{\epsilon}\right) Q\left(\frac{y_{d}}{\epsilon}, \frac{y_{h}}{\epsilon}\right)+F_{d}(t) \Delta+F_{p}(t) \Delta \cos x\right], \\
Q_{2}= & F_{2}-M_{21} M_{11}^{-1} F_{1} \\
& {\left[\begin{array}{l}
\left.\beta \Delta \cos x \dot{x}^{2}-\pi_{h} \dot{y}_{h}-q_{h} \epsilon \frac{y_{h}}{\epsilon}-q_{d} \epsilon \frac{y_{h}}{\epsilon} Q\left(\frac{y_{d}}{\epsilon}, \frac{y_{h}}{\epsilon}\right)-a_{h} \epsilon^{3}\left(\frac{y_{h}}{\epsilon}\right)^{3}+(1+\beta) \Delta\right] \\
+F_{h}(t) \Delta-F_{p}(t) \Delta \sin x-\frac{\beta}{\Delta} \pi_{p} \sin x \dot{x}-\beta \Delta \sin ^{2} x+\beta \Delta \sin x G_{p}(t) \\
\beta \Delta \sin x \dot{x}^{2}-\pi_{d} \dot{y}_{d}-q_{d}\left(1+\epsilon \frac{y_{d}}{\epsilon}\right) Q\left(\frac{y_{d}}{\epsilon}, \frac{y_{h}}{\epsilon}\right)+F_{d}(t) \Delta+F_{p}(t) \Delta \cos x \\
+\frac{\beta}{\Delta} \pi_{p} \cos x \dot{x}+\beta \Delta \sin x \cos x-\beta \Delta \cos x G_{p}(t)
\end{array}\right] . }
\end{aligned}
$$


We therefore obtain

$$
\begin{aligned}
P_{1}(x, v, \eta, w, t ; \epsilon)= & \frac{1+\beta}{\Delta^{2}}\left[-\pi_{p} v-\Delta^{2} \sin x+\Delta^{2} G_{p}(t)+\frac{\Delta}{1+\beta} \sin x\left[\beta \Delta \cos x v^{2}-\pi_{h} w_{h}\right.\right. \\
& \left.-q_{h} \epsilon \eta_{h}-q_{d} \epsilon \eta_{h} Q\left(\eta_{d}, \eta_{h}\right)-a_{h} \epsilon^{3} \eta_{h}^{3}+(1+\beta) \Delta+F_{h}(t) \Delta-F_{p}(t) \Delta \sin x\right] \\
& \left.-\frac{\Delta}{1+\beta} \cos x\left[\beta \Delta \sin x v^{2}-\pi_{d} w_{d}-q_{d}\left(1+\epsilon \eta_{d}\right) Q\left(\eta_{d}, \eta_{h}\right)+F_{d}(t) \Delta+F_{p}(t) \Delta \cos x\right]\right], \\
P_{2}(x, v, \eta, w, t ; \epsilon)= & \epsilon M_{2}^{-1}\left[\begin{array}{l}
\beta \Delta \cos x v^{2}-\pi_{h} w_{h}-q_{h} \epsilon \eta_{h}-q_{d} \epsilon \eta_{h} Q\left(\eta_{d}, \eta_{h}\right)-a_{h} \epsilon^{3} \eta_{h}^{3}+(1+\beta) \Delta \\
+F_{h}(t) \Delta-F_{p}(t) \Delta \sin x-\frac{\beta}{\Delta} \pi_{p} \sin x v-\beta \Delta \sin ^{2} x+\beta \Delta \sin x G_{p}(t) \\
\beta \Delta \sin x v^{2}-\pi_{d} w_{d}-q_{d}\left(1+\epsilon \eta_{d}\right) Q\left(\eta_{d}, \eta_{h}\right)+F_{d}(t) \Delta+F_{p}(t) \Delta \cos x \\
+\frac{\beta}{\Delta} \pi_{p} \cos x v+\beta \Delta \sin x \cos x-\beta \Delta \cos x G_{p}(t)
\end{array}\right],
\end{aligned}
$$

where $M_{2}^{-1}$ is equal to

$$
M_{2}^{-1}=\frac{1}{1+\beta}\left[\begin{array}{cc}
1+\beta \sin ^{2} x & -\beta \sin x \cos x \\
-\beta \sin x \cos x & 1+\beta \cos ^{2} x
\end{array}\right] .
$$

Recall that $\epsilon>0$ has been a completely arbitrary small parameter so far. We now need to define $\epsilon$ in a way that assumptions (A1)-(A3) are satisfied. Since at present we have $\lim _{\epsilon \rightarrow 0} P_{2}(x, v, \eta, w, t ; \epsilon) \equiv$ 0 , these assumptions will not hold. We can only satisfy (A1)-(A3) by making the system parameters appropriate functions of $\epsilon$.

With the parameter choices listed in 62, we have

$$
\begin{aligned}
P_{1}(x, v, \eta, w, t ; \epsilon)= & \frac{1+\beta}{\delta^{2}}\left[-\mu_{p} v-\delta^{2} \sin x+\delta^{2} G_{p}(t)+\frac{\delta}{1+\beta} \sin x\left[\beta \delta \cos x v^{2}-\mu_{h} w_{h}\right.\right. \\
& \left.-\Omega_{h}^{2} \eta_{h}-\Omega_{d}^{2} \eta_{h} Q\left(\eta_{d}, \eta_{h}\right)-\alpha_{h} \eta_{h}^{3}+(1+\beta) \delta+F_{h}(t) \delta-F_{p}(t) \delta \sin x\right] \\
& \left.-\frac{\delta}{1+\beta} \cos x\left[\beta \delta \sin x v^{2}-\mu_{d} w_{d}-\frac{\Omega_{d}^{2}}{\epsilon}\left(1+\epsilon \eta_{d}\right) Q\left(\eta_{d}, \eta_{h}\right)+F_{d}(t) \delta+F_{p}(t) \delta \cos x\right]\right], \\
P_{2}(x, v, \eta, w, t ; \epsilon)= & M_{2}^{-1}\left[\begin{array}{l}
\beta \delta \cos x v^{2}-\mu_{h} w_{h}-\Omega_{h}^{2} \eta_{h}-\Omega_{d}^{2} \eta_{h} Q\left(\eta_{d}, \eta_{h}\right)-\alpha \eta_{h}^{3}+(1+\beta) \delta \\
+F_{h}(t) \delta-F_{p}(t) \delta \sin x-\frac{\beta}{\delta} \mu_{p} \sin x v-\beta \delta \sin ^{2} x+\beta \delta \sin x G_{p}(t) \\
\beta \delta \sin x v^{2}-\mu_{d} w_{d}-\frac{\Omega_{d}^{2}}{\epsilon}\left(1+\epsilon \eta_{d}\right) Q\left(\eta_{d}, \eta_{h}\right)+F_{d}(t) \delta+F_{p}(t) \delta \cos x \\
+\frac{\beta}{\delta} \mu_{p} \cos x v+\beta \delta \sin x \cos x-\beta \delta \cos x G_{p}(t)
\end{array}\right] .
\end{aligned}
$$

where $M_{2}^{-1}$ remains unchanged.

Noting that 


$$
\begin{aligned}
& \lim _{\epsilon \rightarrow 0} \frac{\Omega_{d}^{2}}{\epsilon}\left(1+\epsilon \eta_{d}\right) Q\left(\eta_{d}, \eta_{h}\right) \\
& =\lim _{\epsilon \rightarrow 0} \frac{\Omega_{d}^{2}}{\epsilon}\left(1+\epsilon \eta_{d}\right)\left(1-\frac{1}{\sqrt{\left(1+\epsilon \eta_{d}\right)^{2}+\left(\epsilon \eta_{h}\right)^{2}}}\right) \\
& =\lim _{\epsilon \rightarrow 0} \frac{\Omega_{d}^{2}\left(1+\epsilon \eta_{d}\right)\left(\sqrt{\left(1+\epsilon \eta_{d}\right)^{2}+\left(\epsilon \eta_{h}\right)^{2}}-1\right)}{\epsilon \sqrt{\left(1+\epsilon \eta_{d}\right)^{2}+\left(\epsilon \eta_{h}\right)^{2}}} \\
& =\lim _{\epsilon \rightarrow 0} \frac{f(\epsilon)}{g(\epsilon)}=\lim _{\epsilon \rightarrow 0} \frac{\partial_{\epsilon} f(\epsilon)}{\partial_{\epsilon} g(\epsilon)} \\
& =\lim _{\epsilon \rightarrow 0} \frac{\Omega_{d}^{2} \eta_{d}\left(\sqrt{\left(1+\epsilon \eta_{d}\right)^{2}+\left(\epsilon \eta_{h}\right)^{2}}-1\right)+\Omega_{d}^{2}\left(1+\epsilon \eta_{d}\right)\left(\left(\left(1+\epsilon \eta_{d}\right) \eta_{d}+\epsilon \eta_{h}^{2}\right)\left(\left(1+\epsilon \eta_{d}\right)^{2}+\left(\epsilon \eta_{h}\right)^{2}\right)^{-\frac{1}{2}}\right.}{\sqrt{\left(1+\epsilon \eta_{d}\right)^{2}+\left(\epsilon \eta_{h}\right)^{2}}+\epsilon\left(\left(1+\epsilon \eta_{d}\right) \eta_{d}+\epsilon \eta_{h}^{2}\right)\left(\left(1+\epsilon \eta_{d}\right)^{2}+\left(\epsilon \eta_{h}\right)^{2}\right)^{-\frac{1}{2}}} \\
& =\Omega_{d}^{2} \eta_{d},
\end{aligned}
$$

we conclude that both $P_{1}$ and $P_{2}$ continue to be smooth in $\epsilon$ at the $\epsilon=0$ limit, thereby satisfying assumption (A1).

For the critical manifold defined through the relationship $\eta=G_{0}(x, v, t)$ in assumption (A2), we have the equations

$$
P_{2}(x, v, \eta, 0, t ; 0)=M_{2}^{-1}\left[\begin{array}{l}
\beta \delta \cos x v^{2}-\Omega_{h}^{2} \eta_{h}-\alpha_{h} \eta_{h}^{3}+(1+\beta) \delta+F_{h}(t) \delta \\
-F_{p}(t) \delta \sin x-\frac{\beta}{\delta} \mu_{p} \sin x v-\beta \delta \sin ^{2} x+\beta \delta \sin x G_{p}(t) \\
\beta \delta \sin x v^{2}-\Omega_{d}^{2} \eta_{d}+F_{d}(t) \delta+F_{p}(t) \delta \cos x \\
+\frac{\beta}{\delta} \mu_{p} \cos x v+\beta \delta \sin x \cos x-\beta \delta \cos x G_{p}(t)
\end{array}\right]=\left[\begin{array}{c}
0 \\
0
\end{array}\right] .
$$

Since $M_{2}^{-1}$ is invertible, the critical manifold can be found by solving the following equations for $\eta_{h}$ and $\eta_{d}$ :

$\Omega_{h}^{2} \eta_{h}+\alpha_{h} \eta_{h}^{3}=T_{h}(x, v, t)=\beta \delta \cos x v^{2}+(1+\beta) \delta+F_{h}(t) \delta-F_{p}(t) \delta \sin x-\frac{\beta}{\delta} \mu_{p} \sin x v-\beta \delta \sin ^{2} x+\beta \delta \sin x G_{p}(t)$,

$\Omega_{d}^{2} \eta_{d}=T_{d}(x, v, t)=\beta \delta \sin x v^{2}+F_{d}(t) \delta+F_{p}(t) \delta \cos x+\frac{\beta}{\delta} \mu_{p} \cos x v+\beta \delta \sin x \cos x-\beta \delta \cos x G_{p}(t)$.

The real roots of these two equations can be expressed explicitly as

$$
\begin{gathered}
\eta_{h}=\sqrt[3]{\frac{T_{h}(x, v, t)}{2 \alpha_{h}}+\sqrt{\frac{T_{h}^{2}(x, v, t)}{4 \alpha_{h}^{2}}+\frac{\Omega_{h}^{6}}{27 \alpha_{h}^{3}}}}-\sqrt[3]{-\frac{T_{h}(x, v, t)}{2 \alpha}+\sqrt{\frac{T_{h}^{2}(x, v, t)}{4 \alpha_{h}^{2}}+\frac{\Omega_{h}^{6}}{27 \alpha_{h}^{3}}}}, \\
\eta_{d}=\frac{T_{d}(x, v, t)}{\Omega_{d}^{2}},
\end{gathered}
$$

assuming that $\Omega_{h}^{2}, \Omega_{d}^{2}$ and $\alpha_{h}$ are greater than zero. The stability of this critical manifold is determined by the associated oscillatory system (9), whose coefficient matrices now take the specific 
form

$$
\begin{aligned}
A(x, v, t) & =-\partial_{w} P_{2}\left(x, v, G_{0}(x, v, t), 0, t ; 0\right) \\
& =\frac{1}{1+\beta}\left[\begin{array}{cc}
1+\beta \sin ^{2} x & -\beta \sin x \cos x \\
-\beta \sin x \cos x & 1+\beta \cos ^{2} x
\end{array}\right]\left[\begin{array}{cc}
\mu_{h} & 0 \\
0 & \mu_{d}
\end{array}\right], \\
B(x, v, t) & =-\partial_{\eta} P_{2}\left(x, v, G_{0}(x, v, t), 0, t ; 0\right) \\
& =\frac{1}{1+\beta}\left[\begin{array}{cc}
1+\beta \sin ^{2} x & -\beta \sin x \cos x \\
-\beta \sin x \cos x & 1+\beta \cos ^{2} x
\end{array}\right]\left[\begin{array}{cc}
\Omega_{h}^{2}+3 \alpha_{h} \eta_{h}^{2} & 0 \\
0 & \Omega_{d}^{2}
\end{array}\right] .
\end{aligned}
$$

Consequently, the equilibrium solution of the unforced linear oscillatory system $(9)$ is always asymptotically stable, given that

$$
\mu_{h}>0, \quad \mu_{d}>0 \quad \beta>0, \quad \Omega_{h}^{2}>0, \quad \Omega_{d}^{2}>0, \quad \alpha_{h}>0 .
$$

We conclude that assumptions (A1)-(A3) hold, and hence a global reduced-order model exists over the flexible variables $(x, v, t) \in \mathcal{D}_{0}=\mathbb{R} \times \mathbb{R} \times S^{1}$.

\section{References}

[1] Ariel, G., Sanz-Serna, J. M., and Tsai, R., A multiscale technique for finding slow manifolds of stiff mechanical systems. Musltiscale Model. Simul. 10 (2012) 1180-1203.

[2] Arnold, V.I., Catastrophe Theory, 3rd ed. Berlin: Springer-Verlag (1992).

[3] Benner, P., Gugwrcin, S., Willcox, K, A survey of projection-based model reduction methods for parametric dynamical systems. SIAM Review. 57 (2015)483-53.

[4] Besselink, B., Tabak, U., Lutowska, A., van de Wouw, N., Nijmeijer, H., Rixen, D.J., Hochstenbach, M.E. and Schilders, W.H.A., A comparison of model reduction techniques from structural dynamics, numerical mathematics and systems and control. J. Sound Vibration 332 (2013) 4403-4422.

[5] Cabré, P., Fontich, E., and de la Llave, R., The parametrization method for invariant manifolds I: Manifolds associated to non-resonant spectral subspaces. Indiana University Mathematics J. 52 (2003) 283-328.

[6] Carr, J., Applications of Centre Manifold Theory. Springer, New York (1982).

[7] Cheong, J., Cho, Y., and Lee, S.I., Invariant slow manifold approach to exact dynamics inversion of singularly perturbed linear mechanical systems with admissible output constraints. J. Sound and Vibration 331 (2012) 3710-3720.

[8] Fenichel, N., Geometric singular perturbation theory for ordinary differential equations. J. Diff. Eqs. 31 (1979) 53-98.

[9] Georgiou, I.T., Bajaj, A.K., and Corless, M., Invariant manifolds and chaotic vibrations in singularly perturbed nonlinear oscillators. Int. J. Engng. Sci. 36 (1998) 431-458.

[10] Georgiou, I.T., and Schwartz, I.B., The slow invariant manifold of a conservative pendulumoscillator system. Int. J. Bifurcation and Chaos 6 (1996) 673-692.

[11] Georgiou, I.T., and Vakakis, A.F., An invariant manifold approach for studying waves in a onedimensional array of non-linear oscillators. Int. J. Non-Linear Mechanics. (31) (1996) 871-886.

[12] Georgiou, I.T., Corless, M.J., and Bajaj, A.K., Dynamics of nonlinear structures with multiple equilibria: A singular perturbation-invariant manifold approach. Z. Angew. Math. Phys. 50 (1999) 892-924. 
[13] Georgiou, I.T., and Schwartz, I.B., Dynamics of large scale coupled structural/mechanical systems: A singular perturbation/proper orthogonal decomposition approach. SIAM J. App. Math. 59 (1999) 1178-1207.

[14] Geradin, M., and Rixan, D.J., Mechanical Vibrations: Theory and Application to Structural Dynamics, (3rd ed). Wiley (2015).

[15] Guckenheimer, J. and Holmes, P.J., Nonlinear Oscillations, Dynamical Systems and Bifurcations of Vecotr fields. Springer, New York (1983).

[16] Guyan, R.J., Reduction of stiffness and mass matrices. AIAA Journal 3 (1965) p. 380.

[17] Haller, G. and Ponsioen, S., Nonlinear normal and spectral submanifolds: Existence, uniqueness and use in model reduction. Nonlinear Dynamics, in press (2016).

[18] Idelsohn, S.R., and Cardona, A., A reduction method for nonlinear structural dynamic analysis. Comput. Methods Appl. Mech. Eng. 49(3) (1985) 253-279.

[19] Jain, S., Tiso, P., and Haller, G., Exact nonlinear model reduction by Slow-Fast Decomposition for a forced von Kármán beam, preprint (2016).

[20] Jones, C.K.R.T., Geometric singular perturbation theory, in Dynamical Systems, Lecture Notes in Mathematics 1609, Springer, New York (2006) 44-11.

[21] Kelley, A. F., Analytic two-dimensional subcenter manifolds for systems with an integral. Pacific J. of Mathematics. 29 (1969) 335-350.

[22] Kristiansen, K.U., and Wulff, C., Exponential estimates of symplectic slow manifolds, J. Diff. Eqs. 261 (2016) 56-101.

[23] Kurt, M., Eriten, M., McFarland, D.M., Bergman, L. A., and Vakakis, A. F., Strongly nonlinear beats in the dynamics of an elastic system with a strong local stiffness nonlinearity: Analysis and identification. J. of Sound and Vibration 333 (2014) 2054-2072.

[24] Lubich, C., Integration of stiff mechanical systems by Runge-Kutta methods, Z. Angew. Math. Phys. 44 (1993) 1022-1053.

[25] MacKay, R.S., Slow manifolds. In: Energy Localisation and Transfer, eds.: T. Dauxois, A. Litvak-Hinenzon, RS MacKay, A Spanoudaki, World Scientific (2004) 149-192.

[26] Menon, G., and Haller, G., Infinite-dimensional geometric singular perturbation theory for the Maxwell-Bloch equations. SIAM J. Math. Anal. 33 (2001) 315-346.

[27] Mignolet, M.P., Przekop, A., Rizzi, S.A., and Spottswood, S.M., A review of indirect/nonintrusive reduced order modeling of nonlinear geometric structures. J. Sound and Vibration 332 (2013) 2437-2460.

[28] Rutzmoser, J.B., Rixen, D.J., and Tiso, P., Model order reduction using an adaptive basis for geometrically nonlinear structural dynamics. in Proc. Int. Conf. on Noise and Vibration Engineering, ISMA (2014).

[29] Shaw, S. W., and Pierre, C., Normal modes for non-linear vibratory systems. J. Sound and Vibrations 164 (1993) 85-124.

[30] Sombroek, C., Renson, L., Tiso, P., and Kerschen, G., Bridging the gap between nonlinear normal modes and modal derivatives, in Nonlinear Dynamics, Volume 1, Proceedings of the 33rd IMAC, A Conference and Exposition on Structural Dynamics, 2015. (G. Kerschen (ed.)), Springer (2016) 349-361. 
[31] Stumpp, T., Asymptotic expansions and attractive invariant manifolds of strongly damped mechanical systems, ZAMM - Z. Angew. Math. Mech. 88 (2008) 630-643.

[32] Vakakis, A. F., Gendelman, O.V., Bergman, L.A., McFarland, D.M., Kerszhen, G., and Lee, Y.S., Nonlinear Targeted Energy Transfer in Mechanical and Structural Systems. Springer Science+Business Media, New York (2008)

[33] Wu, L. and Tiso, P., Nonlinear model order reduction for flexible multibody dynamics: a modal derivatives approach. Multibody Syst. Dyn. 36 (2016) 405-425. 